\title{
A Network Diffusion Model of Food Safety Scare Behavior considering Information Transparency
}

\author{
Tingqiang Chen, ${ }^{1,2}$ Lei Wang, ${ }^{1}$ Jining Wang, ${ }^{1}$ and Qi Yang ${ }^{1}$ \\ ${ }^{1}$ School of Economics and Management, Nanjing Tech University, Nanjing 211816, China \\ ${ }^{2}$ School of Management Science and Engineering, Nanjing University, Nanjing 210093, China \\ Correspondence should be addressed to Jining Wang; wangjn163@126.com and Qi Yang; yq@njtech.edu.cn
}

Received 16 June 2017; Revised 14 November 2017; Accepted 23 November 2017; Published 21 December 2017

Academic Editor: Danilo Comminiello

Copyright (c) 2017 Tingqiang Chen et al. This is an open access article distributed under the Creative Commons Attribution License, which permits unrestricted use, distribution, and reproduction in any medium, provided the original work is properly cited.

\begin{abstract}
This study constructs the network diffusion model of food safety scare behavior under the effect of information transparency and examines the network topology and evolution characteristics of food safety scare behavior in a numerical simulation. The main conclusions of this study are as follows. (1) Under the effect of information transparency, the network degree distribution of food safety scare behavior diffusion demonstrates the decreasing characteristics of diminishing margins. (2) Food safety scare behavior diffusion increases with the information dissemination rate and consumer concern about food safety incidents and shows the characteristics of monotone increasing. And with the increasing of the government food safety supervision information transparency and media food safety supervision information transparency, the whole is declining characteristic of diminishing marginal. In addition, the extinction of food safety scare behavior cannot be achieved gradually given a single regulation of government food safety supervision information transparency and media food safety supervision information transparency. (3) The interaction effects between improving government food safety supervision information transparency or media food safety supervision information transparency and declining consumer concerns about food safety incidents or information transmission rate can engender the suppression of food safety scare behavior diffusion.
\end{abstract}

\section{Introduction}

Food safety problem is given attention by governments and academics worldwide [1]. The losses caused by social panic are more than the direct losses of food safety incidents $[2$, 3] and have a serious impact on social stability and the development of the food industry [4].

Food safety scare behavior refers to the anxiety of consumers in a situation of information asymmetry. The combining research on food safety and consumer behavior mainly focuses on consumer concerns about food safety [57], purchase intention for safe food [8-12], and the risk perception of food safety [13-17]. The idea that food safety information transparency eases food safety issues has been given increasing attention by scholars globally with the further development of food safety management. The analysis of the food safety management system and the appropriate information disclosure system can effectively control food safety [18]. Relying only on the market economy mechanism hardly guarantees food safety owing to the public nature of food safety and food risk information asymmetry [19]. Therefore, in order to overcome the deficiency of adjusting food safety problem only depending on market economy mechanism, the government food safety supervision department, which is the main provider of food safety supervision information and the most important subject of food safety supervise, should unite media and other subjects of social supervision to achieve food safety supervision information transparency [20, 21]. However, the research on the diffusion mechanisms and the evolution characteristics of food safety scare behavior are rare and not conducive to control and reduce the losses caused by food safety scare behavior diffusion. The present study, hence, attempts to introduce information transparency into the study of food safety scare behavior diffusion and analyze the evolution characteristics of food safety scare behavior diffusion under the effect of food safety supervision information transparency. 
At present, the epidemic model was proposed based on the complex network theory, which has been widely used in various fields [12, 20-23]. Studies on the epidemic model have been extensively used in biological and ecological applications [24-26]. The epidemic model is being gradually extended to the field of Social Science, such as in technology and innovation diffusion (Sven \& Johannes, 2013) [27], financial risk contagion [28-30], and rumor spreading $[31,32]$. These studies provide the necessary technological means for solving social problems. Developing the epidemic model can provide insight into the diffusion mechanism of infectious diseases and provide theoretical basis for devising a coupling strategy. Food safety scare behavior caused by food safety incidents can spread through a certain medium to healthy consumers [12]. Therefore, food safety scare behavior is a typical diffusion problem, and similar mechanisms exist with the spread of infectious diseases. Moreover, the existing empirical study results confirm that improving food safety supervision information transparency can reduce the adverse effects caused by food safety incidents [20,21]. The present study, thus, uses the epidemic model for constructing the network diffusion model of food safety scare behavior under the effect of the information transparency, analyzes the diffusion mechanisms of different information transparency of food safety scare behavior, and provides a reference for the control of the adverse social impact of food safety scare behavior diffusion.

The structure of this study is organized as follows. Section 2 analyzes the epidemic mechanisms of food safety scare behavior under the effect of information transparency. Section 3 constructs the network diffusion model of food safety scare behavior under different information transparency. Section 4 theoretically analyzes the network topology characteristics of the food safety scare behavior under the effect of information transparency. Section 5 discusses the network topology characteristics and evolution characteristics of food safety scare behavior under different information transparency and provides strategies for controlling food safety scare behavior diffusion. Section 6 concludes this paper.

\section{Epidemic Mechanisms of Food Safety Scare Behavior Diffusion under the Effect of Information Transparency}

\subsection{Adaptability Analysis of Epidemic Model of Food Safety Scare Behavior Diffusion. Epidemic model is a classic virus propagation model and has been widely used in the study of social behavior diffusion [33-36]. The essence of an infectious disease is a virus carrier, a pathogen, which infects its own virus to contacts through a certain medium [37]. Food safety scare behavior diffusion means that consumers' food safety scare behavior spreads to contacts through various diffusion media. Food safety scare behavior is a virus that affects the population, and similar mechanisms exist in the process of spreading. The principal representations are as follows.}

(1) Pathogen-Diffusion Source. Food safety scare behavior diffusion is mainly due to the public's concern about food safety issues [38]. Diffusion source means that consumers' food safety scare behavior can spread among consumers through diffusion media, which results in a significant herd effect.

(2) Infectious Medium-Diffusion Medium. A diffusion medium is a carrier of the diffusion source, such as the Internet, mobile phones, TVs, and face-to-face communication between consumers. Food safety information spread by diffusion media is related to the health and life safety of consumers, and the transparency of food safety information affects consumers' confidence in food safety $[20,21]$.

(3) Infectiousness. Consumers affected by food safety scare transfer the information of their cognitive psychology and behavioral deviations to healthy consumers through diffusion media under the effect of information transparency. This transfer of information shows consumers' concern degree and cognitive psychology deviation that engender scare behavior. Therefore, food safety scare behavior is contagious. Under information transparency, consumers who are affected by food safety scare spread their own mental state, behavioral deviation, and other information through kinship and work relationships to influence consumers in their healthy state, thereby engendering food safety scare behavior (Figure 1).

(4) Immunity. Consumers can obtain adequate information on food safety after the outbreak of food safety incidents when food safety information transparency is high $[5,39]$ and, thus, have a clear understanding of the food safety incidents. Consequently, food safety scare behavior hardly affects such consumers. This type of consumers shows an immune response to food safety scare behavior. However, consumers have difficulty in obtaining adequate food safety information to identify rumors about food safety incidents when food safety information transparency is low. Therefore, food safety scare behavior easily affects such consumers.

The spread of food safety scare behavior diffusion has a similar epidemic mechanism under the effect of information transparency. However, the subjects of food safety supervision information are complex and diverse, and many interests in the process of transparency are driven. Accordingly, food safety scare behavior in the diffusion process is more complex compared with the spread of the virus. Therefore, using the epidemic model to analyze the diffusion mechanisms and the evolution characteristics of different information transparency of food safety scare behavior is scientific and feasible. Using this model can provide a reference for the control of food safety scare behavior diffusion. Table 1 shows that the key concepts in the epidemic model are transferred to food safety scare behavior diffusion.

2.2. Diffusion Mechanism of Food Safety Scare Behavior. Market information is distorted after the outbreak of food safety incidents. When information transparency is low, consumers who have a low cognitive level, weak psychological quality, 


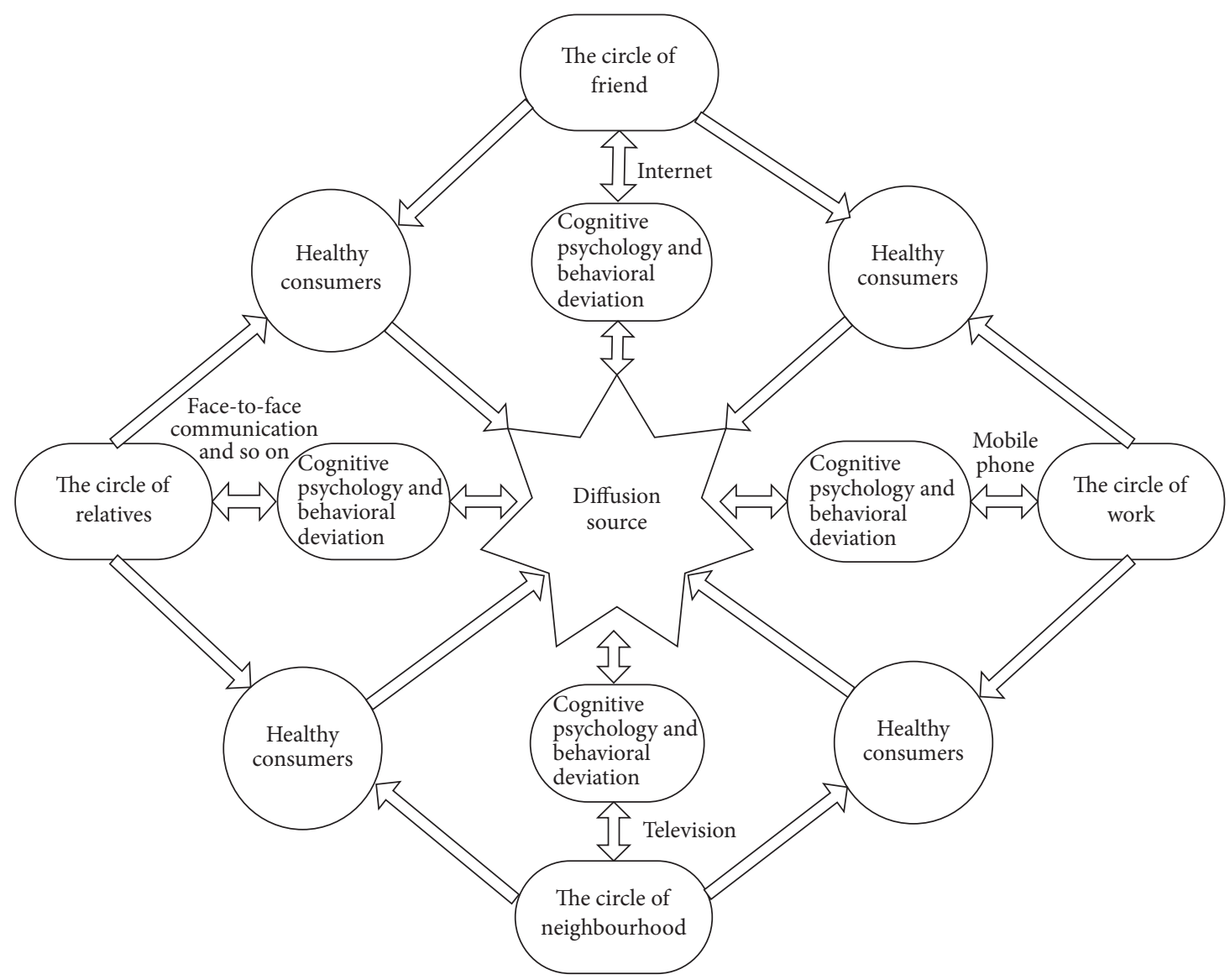

FIGURE 1: Diffusion medium and diffusion path of food safety scare behavior.

TABLE 1: Corresponding concept of food safety scare behavior diffusion.

Food safety scare behavior diffusion Meaning

Diffusion source

Healthy consumers

Infected consumers

Immune consumers

Diffusion rate

Immunization rate
Consumers' food safety scare behavior

Consumers are not affected by food safety scare behavior

Consumers influenced by diffusion source are affected by food safety scare behavior

Consumers who are not affected or have been affected by food safety scare behavior get rid of this behavior through adjustment

Consumers affected food safety scare behavior in the proportion of healthy consumers

Consumers who are not affected by food safety scare behavior or those who have been affected and then got rid of this behavior through adjustment in the proportion of healthy consumers and poor information search ability cannot fully perceive and discriminate the real effect of food safety incidents and have an objective understanding of the food safety incidents, which lead to cognitive psychology and behavioral deviations $[20,21,40,41]$. Then, this can make consumers confuse major food safety accidents with ordinary food events and make it easy to initiate consumers' general panic to food safety. Some consumers have food safety scare behavior, whereas others are in a state of potential panic. Consumers who have food safety scare behavior spread their own mental state and behavioral deviation and other information to the outside environment through kinship and work relationships and influence healthy consumers. Furthermore, food safety scare behavior may also be transferred to consumers who have poor psychological quality and lack food safety information [42]. Consumers with food safety scare behavior can get rid of food safety scare because of the improvement of food safety knowledge and the increase of market objective information. Therefore, consumers are divided into three states after the outbreak of food safety incidents. $S$ represents the number of consumers who do not have food safety scare behavior: the health state. $I$ represents the number of consumers with food safety scare behavior: the infection state. $R$ represents the number of consumers who are not affected by food safety scare behavior or those who have been affected and then got rid of this behavior through adjustment: the immune state. 


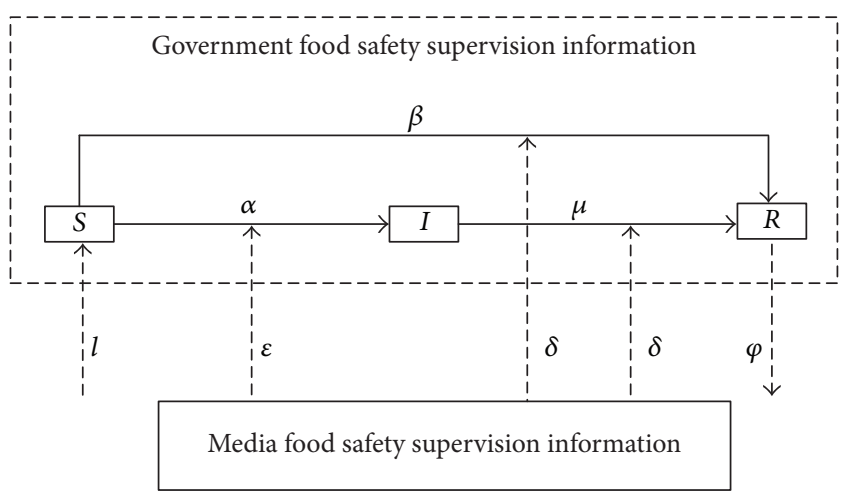

Figure 2: Diffusion model of food safety scare behavior under the effect of information transparency.

The transition of consumers in the health state $S$, infection state $I$, and immune state $R$ follows the following diffusion rules (Figure 2).

(1) After the outbreak of food safety incidents, if the supervision information transparency issued by the government supervision department is low, then consumers' food safety scare behavior diffuses to healthy consumers at the rate of $\alpha(0 \leq \alpha \leq 1)$. Moreover, if the food safety supervision information transparency reported by the media is low, then consumers' food safety scare behavior further diffuses to healthy consumers at the rate of $\varepsilon(0 \leq \varepsilon \leq 1)$.

(2) Some consumers have strong psychological qualities and knowledge of food safety; hence, they are free of food safety scare behavior and are in the immune state $R$ with the probability of $\beta(0 \leq \beta \leq 1)$. The media have a high transparency of the information on food safety supervision, which prompted some health consumers to be directed into the immune state $R$ with the probability of $\delta(0 \leq \delta \leq 1)$.

(3) If the government supervision department on food safety supervision information is transparent, then consumers with food safety scare behavior can get rid of food safety scare behavior and be directed into the immune state with the probability of $\mu(0 \leq \mu \leq 1)$. Furthermore, if the food safety supervision information transparency reported by media is high, then consumers with food safety scare behavior can get rid of food safety scare behavior and be directed into the immune state with the probability of $\delta$.

(4) In each period, the rate of entry for consumers is $l(0 \leq$ $l \leq 1)$ and the exit rate is $\varphi(0 \leq \varphi \leq 1)$.

\section{Network Diffusion Model of Food Safety Scare Behavior under Different Information Transparency}

3.1. Constructing Model. To construct the network diffusion model of food safety scare behavior, we assume $N$ as the total number of consumers in food safety incidents. $s, i, r$ represent the proportion of healthy, infected, and immune consumers, respectively: $s=S / N, i=I / N, r=R / N$, and $s+i+r=$ $1(0 \leq s, i, r \leq 1)$. The density of the infected consumers, whose degree of $k$ is $i_{k}(t)$ at the moment $t$, is assumed. The probability that the healthy consumers are connected with the infected consumers is $\Theta(t)$.

Market information has a certain impact on individual behavior; thus, Gilpin and Ayala [43] define the information diffusion model.

$$
f(Q(t))=\lambda Q\left[1-\left(\frac{Q}{M}\right)^{\gamma}\right],
$$

where $Q$ represents the number of individuals with information in the process of information diffusion, $\lambda$ is the rate of information dissemination, which represents the ability of each individual with information to transmit information $(0<\lambda \leq 1), M$ represents the total number of individuals in a population and is a constant, and $\gamma$ represents the public's sensitivity to events $(0<\gamma \leq 1)$.

The Gilpin-Ayala information diffusion model states that if factors that affect the information transparency of food safety scare behavior diffusion, which includes government food safety supervision information transparency [20,21] and consumer concerns about food safety incidents [5-7], are combined, then $\alpha$ is defined as follows:

$$
\alpha=\lambda\left(1-e^{-\theta / w}\right)
$$

where $\lambda$ represents the rate of information dissemination $(0<$ $\lambda \leq 1)$, $w$ represents government food safety supervision information transparency $(0<w \leq 1)$, and $\theta$ represents consumer concerns about food safety incidents $(0<\theta \leq 1)$.

The Gilpin-Ayala information diffusion model is used with the effect of food safety scare behavior information transparency factors, which include media food safety supervision information transparency $[20,21]$ and consumer concerns about food safety incidents [5-7]; $\varepsilon$ is defined as follows:

$$
\varepsilon=\lambda\left(1-e^{-\theta / h}\right),
$$

where $\lambda$ and $\theta$ have the same definitions of the diffusion rate $\alpha$ and $h$ represents media food safety supervision information transparency $(0<h \leq 1)$.

Based on mean field theory [44-47] and the abovementioned hypothesis, the differential equations of the network diffusion model of food safety scare behavior under the effect of information transparency are

$$
\begin{aligned}
& \frac{d s_{k}(t)}{d t}=l-k(\alpha+\varepsilon) s_{k}(t) \Theta(t)-(\beta+\delta) s_{k}(t), \\
& \frac{d i_{k}(t)}{d t}=k(\alpha+\varepsilon) s_{k}(t) \Theta(t)-(\mu+\delta) i_{k}(t), \\
& \frac{d r_{k}(t)}{d t}=(\mu+\delta) i_{k}(t)+(\beta+\delta) s_{k}(t)-\varphi r_{k}(t) .
\end{aligned}
$$

3.2. Analyzing Model. According to (4), for the steady-state condition $d i_{k}(t) / d t=0$, the steady-state value becomes $i_{k}(t)$.

$$
\begin{aligned}
i_{k}(t) & =\frac{k(\alpha+\varepsilon) s_{k}(t) \Theta(t)}{\mu+\delta} \\
& =\frac{k l(\alpha+\varepsilon) \Theta(t)}{(\beta+\delta)(\mu+\delta)+k(\alpha+\varepsilon)(\mu+\delta) \Theta(t)} .
\end{aligned}
$$


The average infected consumer density becomes $i=$ $\sum_{k} P(k) i_{k}(t)$. Based on formula (5), $\Theta(t)$ becomes

$$
\Theta(t)=\sum_{k} \frac{k P(k) i_{k}(t)}{\sum_{s} s P(s)}=\frac{1}{\langle k\rangle} \sum_{k} k P(k) i_{k}(t),
$$

where $\langle k\rangle$ represents the average degree of food safety scare behavior diffusion.

Given that $\langle k\rangle=\sum_{k} k P(k)$ and $\left\langle k^{2}\right\rangle=\sum_{k} k^{2} P(k),(5)$ and (6) can be combined as follows:

$$
\begin{aligned}
\Theta(t) & =\frac{1}{\langle k\rangle} \sum_{k} k P(k) \\
\cdot & \frac{k l(\alpha+\varepsilon) \Theta(t)}{(\beta+\delta)(\mu+\delta)+k(\alpha+\varepsilon)(\mu+\delta) \Theta(t)} .
\end{aligned}
$$

Given that $\Theta=\Theta(t),(7)$ has a trivial solution: $\Theta=0$. If (7) has a nontrivial solution, $\Theta \neq 0$, then the necessary condition becomes

$$
\begin{aligned}
& \frac{d}{d \Theta}\left(\frac{1}{\langle k\rangle} \sum_{k} k P(k)\right. \\
& \left.\cdot \frac{k l(\alpha+\varepsilon) \Theta}{(\beta+\delta)(\mu+\delta)+k(\alpha+\varepsilon)(\mu+\delta) \Theta}\right)\left.\right|_{\Theta=0} \geq 1 .
\end{aligned}
$$

Therefore,

$$
\frac{1}{\langle k\rangle} \sum_{k} k P(k) \frac{k l(\alpha+\varepsilon)}{(\beta+\delta)(\mu+\delta)} \geq 1 .
$$

Thus, the basic reproduction number of food safety scare behavior diffusion under different information transparency is $R_{0}$ (the basic reproduction number indicates that the average number of individuals who are susceptible to infection before infection is recovered [48]. $R_{0}=1$ corresponds to the threshold of the extinction of the diffusion. The diffusion becomes extinct gradually when $R_{0}<1$. The diffusion occurs with nonzero probability when $R_{0}>1$. The greater the value of $R_{0}$ is, the greater the probability of diffusion becomes).

$$
\begin{aligned}
R_{0} & =\frac{l \sum_{k} k^{2} P(k)(\alpha+\varepsilon)}{(\beta+\delta)(\mu+\delta) \sum_{k} k P(k)} \\
& =\frac{\lambda l\left(2-e^{-\theta / w}-e^{-\theta / h}\right) \sum_{k} k^{2} P(k)}{(\beta+\delta)(\mu+\delta) \sum_{k} k P(k)} .
\end{aligned}
$$

Equation (10) shows that the basic reproduction number is obtained by acquiring the degree distribution function $P(k)$ of food safety scare behavior under the effect of information transparency.

\section{Network Topology Characteristics of Food Safety Scare Behavior under Different Information Transparency}

The node in the food safety scare behavior diffusion network represents the consumer in the food safety scare behavior diffusion. Two consumers are connected to the side. The algorithm is described as follows.

(1) $m_{0}$ consumers with food safety scare behavior and $n_{0}$ sides $\left(m_{0}>0, n>0\right)$ exist at $t_{0}$.

(2) At each period $t_{i}(i=1,2,3, \ldots), m$ consumers with food safety scare behavior are increased in the network, and every new consumer has $\eta$ sides $(m>0, \eta>0)$.

(3) Without considering the factors of information transparency, the new consumers connect the consumer with food safety scare behavior randomly in the proportion of $p$ or connect the consumer with food safety scare behavior preferentially in the proportion of $(1-p)(0 \leq p \leq 1)$. When information transparency is introduced, the probability of random connection is

$$
p^{*}=p^{w h /(\lambda \theta)^{1 / 2}} .
$$

(4) The probability that any existing consumer $i$ is selected in random linking is $1 /\left(m_{0}+m t\right)$. The probability that any existing consumer $i$ is selected in preferential linking is $\Pi_{i}\left(0 \leq \Pi_{i} \leq 1\right)$.

$$
\Pi_{i}=\frac{k_{i}}{\sum_{j} k_{j}}
$$

where $k_{i}$ represents the degree of existing consumer $i$.

The above algorithm shows that the change rate of degree $k_{i}$ of consumer $i$ can be expressed as follows:

$$
\begin{aligned}
\frac{\partial k_{i}}{\partial t} & =\frac{m \eta p^{*}}{m_{0}+m t}+\left(1-p^{*}\right) m \eta \Pi_{i} \\
& =\frac{m \eta p^{*}}{m_{0}+m t}+\left(1-p^{*}\right) m \eta \frac{k_{i}}{\sum_{j} k_{j}} .
\end{aligned}
$$

Given that $\sum_{j} k_{j}=2\left(m \eta t+n_{0}\right),(13)$ can be translated into the following:

$$
\frac{\partial k_{i}}{\partial t}=\frac{m \eta p^{*}}{m_{0}+m t}+\left(1-p^{*}\right) m \eta \frac{k_{i}}{2\left(m \eta t+n_{0}\right)},
$$

where $t \rightarrow \infty, m t+m_{0} \approx m t$, and $m \eta t+n_{0} \approx m \eta t$. The initial condition can obtain $k_{j}\left(t_{j}\right)=m \eta$. Therefore, the solution of (14) is

$$
k_{i}=\left(m \eta+\frac{2 \eta p^{*}}{1-p^{*}}\right)\left(\frac{t}{t_{i}}\right)^{\left(1-p^{*}\right) / 2}-\frac{2 \eta p^{*}}{1-p^{*}} .
$$

When consumers enter the network at every similar period, the probability density of the selected time node $t_{i}$ is

$$
P_{i}=\frac{1}{m t+m_{0}} .
$$

When $k_{i}<k, P\left(k_{i}(t)<k\right)$ is

$$
\begin{aligned}
& P\left(k_{i}(t)<k\right) \\
& \quad=P\left(t_{i}>t\left[\frac{k\left(1-p^{*}\right)+2 \eta p^{*}}{m \eta\left(1-p^{*}\right)+2 \eta p^{*}}\right]^{-2 /\left(1-p^{*}\right)}\right) \\
& \quad=1-P\left(t_{i} \leq t\left[\frac{k\left(1-p^{*}\right)+2 \eta p^{*}}{m \eta\left(1-p^{*}\right)+2 \eta p^{*}}\right]^{-2 /\left(1-p^{*}\right)}\right) .
\end{aligned}
$$


Equations (16) and (17) are combined to obtain

$$
\begin{aligned}
& P\left(k_{i}(t)<k\right) \\
& \quad=1-\frac{t}{m_{0}+m t}\left[\frac{k\left(1-p^{*}\right)+2 \eta p^{*}}{m \eta\left(1-p^{*}\right)+2 \eta p^{*}}\right]^{-2 /\left(1-p^{*}\right)}, \\
& \lim _{t \rightarrow \infty} P\left(k_{i}(t)<k\right) \\
& \quad \approx 1-\frac{1}{m}\left[\frac{k\left(1-p^{*}\right)+2 \eta p^{*}}{m \eta\left(1-p^{*}\right)+2 \eta p^{*}}\right]^{-2 /\left(1-p^{*}\right)} .
\end{aligned}
$$

Based on (19), the degree distribution function of food safety scare behavior diffusion network is

$$
\begin{aligned}
& P(k)=\frac{\partial P\left(k_{i}(t)<k\right)}{\partial k} \\
& =\frac{2}{m\left[m \eta\left(1-p^{*}\right)+2 \eta p^{*}\right]}\left[\frac{k\left(1-p^{*}\right)+2 \eta p^{*}}{m \eta\left(1-p^{*}\right)+2 \eta p^{*}}\right]^{\left(p^{*}-3\right) /\left(1-p^{*}\right)} .
\end{aligned}
$$

Equation (20) is integrated with (10); hence,

$$
\begin{aligned}
& R_{0}=\frac{\lambda l\left(2-e^{-\theta / w}-e^{-\theta / h}\right) \sum_{k} k^{2} P(k)}{(\beta+\delta)(\mu+\delta) \sum_{k} k P(k)} \approx \frac{\lambda l\left(2-e^{-\theta / w}-e^{-\theta / h}\right) \int_{m \eta}^{\infty} k^{2}\left[k\left(1-p^{*}\right)+2 \eta p^{*}\right]^{\left(p^{*}-3\right) /\left(1-p^{*}\right)} d k}{(\beta+\delta)(\mu+\delta) \int_{m \eta}^{\infty} k\left[k\left(1-p^{*}\right)+2 \eta p^{*}\right]^{\left(p^{*}-3\right) /\left(1-p^{*}\right)} d k} \\
& \approx \frac{\lambda l \eta\left(2-e^{-\theta / w}-e^{-\theta / h}\right)\left[m^{2}-\left(m^{2}-4 m\right) p^{w h /(\lambda \theta)^{1 / 2}}-\left(m^{2}+8 m-4\right) p^{2 w h /(\lambda \theta)^{1 / 2}}+\left(m^{2}+4 m-8\right) p^{3 w h /(\lambda \theta)^{1 / 2}}+4 p^{4 w h /(\lambda \theta)^{1 / 2}}\right]}{2(\beta+\delta)(\mu+\delta)\left[m p^{w h /(\lambda \theta)^{1 / 2}}+(1-2 m) p^{2 w h /(\lambda \theta)^{1 / 2}}+(m-2) p^{3 w h /(\lambda \theta)^{1 / 2}}+p^{4 w h /(\lambda \theta)^{1 / 2}}\right]} .
\end{aligned}
$$

Equation (21) shows that the effect on the rate of information dissemination $\lambda$, consumer concerns about food safety incidents $\theta$, government food safety supervision information transparency $w$, and media food safety supervision information transparency $h$ on the basic reproductive number $R_{0}$ can be analyzed.

\section{Analogue Simulation}

Numerical simulation analysis is the most effective way for testing without a large number of empirical validations of real-time dynamic data [49]. Therefore, we can assume $m=$ $\eta=5$ and $k=1000$ given the different parameters. We can simulate the network topology characteristics and evolution characteristics of food safety scare behavior under the effect of information transparency using the MATLAB R2012b software.

5.1. Analysis of Network Topology Characteristics of Food Safety Scare Behavior under Different Information Transparency. To describe the network topology characteristics of food safety scare behavior under the effect of information transparency with the different rates of information dissemination $\lambda$, consumers concern about food safety incidents $\theta$, government food safety supervision information transparency $w$, and media food safety supervision information transparency $h$, the impact of network topology on the food safety scare behavior diffusion is simulated (Figure 3 ). The initial values are $\theta=0.2, p=\lambda=h=0.3$, and $w=0.4$.

Figure 3 shows that the network degree distribution of food safety scare behavior diffusion under the effect of information transparency shows the decreasing characteristic of diminishing margins. The comparative analysis of Figures 3(a), 3(b), 3(c), and 3(d) shows that the information transmission rate $\lambda$, consumer concerns about food safety incidents $\theta$, government food safety supervision information transparency $w$, and media food safety supervision information transparency $h$ have different effects on the network degree distribution of food safety scare diffusion behavior, whose prominence is as follows: government food safety supervision information transparency $w$ on the network distribution of food safety scare diffusion behavior, consumer concerns about food safety incidents $\theta$ on the network degree distribution of food safety scare behavior diffusion, the rate of information dissemination $\lambda$ on the network degree distribution of food safety scare behavior diffusion, and media food safety supervision information transparency $h$ on the network degree distribution of food safety scare behavior diffusion. Moreover, Figure 3 shows that the sensitivity of network degree distribution of food safety scare behavior diffusion is enhanced along with the increase of the rate of information dissemination $\lambda$ or consumer concerns about food safety incidents $\theta$. The sensitivity of the network degree distribution of food safety scare behavior diffusion reduces along with the increase of government food safety supervision information transparency $w$ or media food safety supervision information transparency $h$.

In order to better describe the influence of the speed of information dissemination, consumers' attention to food safety accidents, the government food safety supervision information transparency, and media food safety supervision information transparency on network topology characteristics of food safety panic behavior diffusion, under the circumstance of $m=\eta=5, k=600$, and $p=0.3$, we conduct sensitivity analysis on $\theta, \lambda, w$, and $h$, as shown in Tables 2 and 3.

Through the sensitivity analysis of Tables 2 and 3, the conclusions obtained in Figure 3 are further verified. And we find that the government food safety supervision information transparency and media food safety supervision information transparency have the "scatter effect" on the network degree distribution of food safety scares behavior diffusion; 

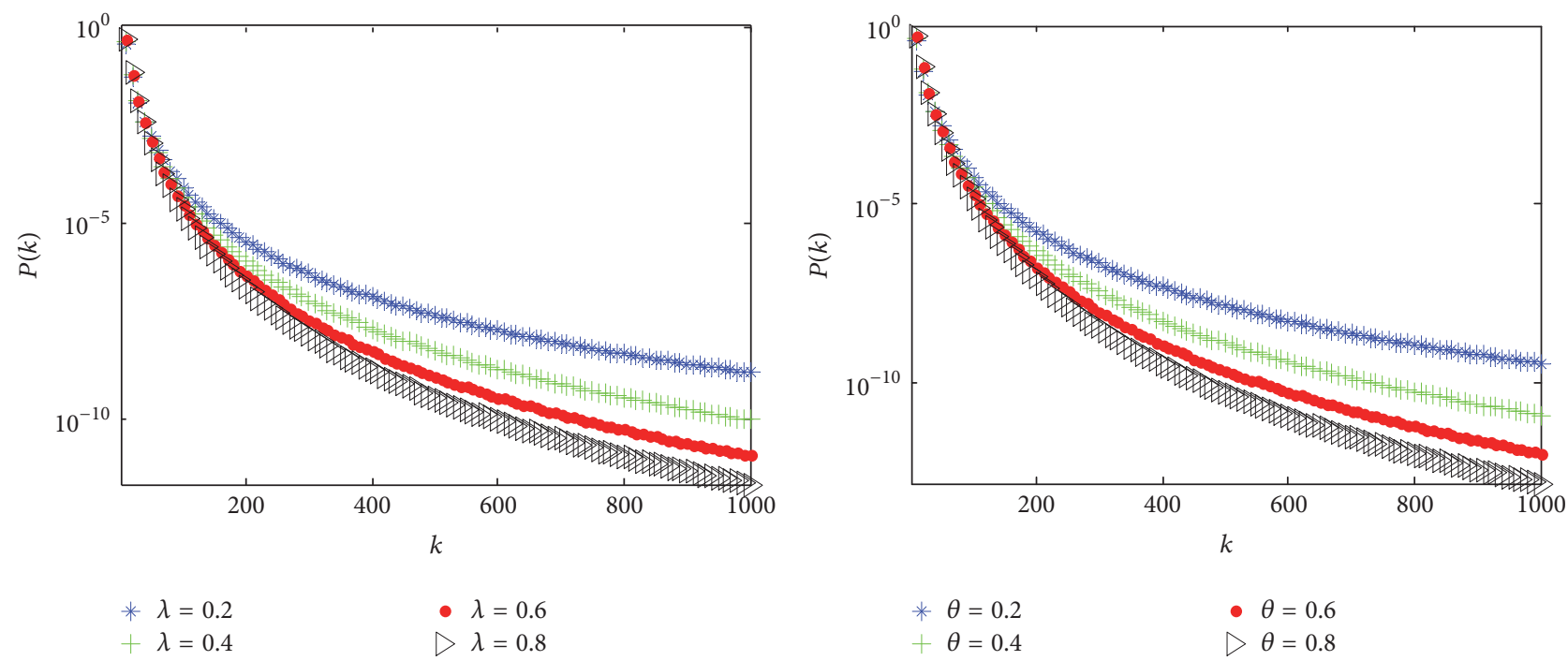

(a)

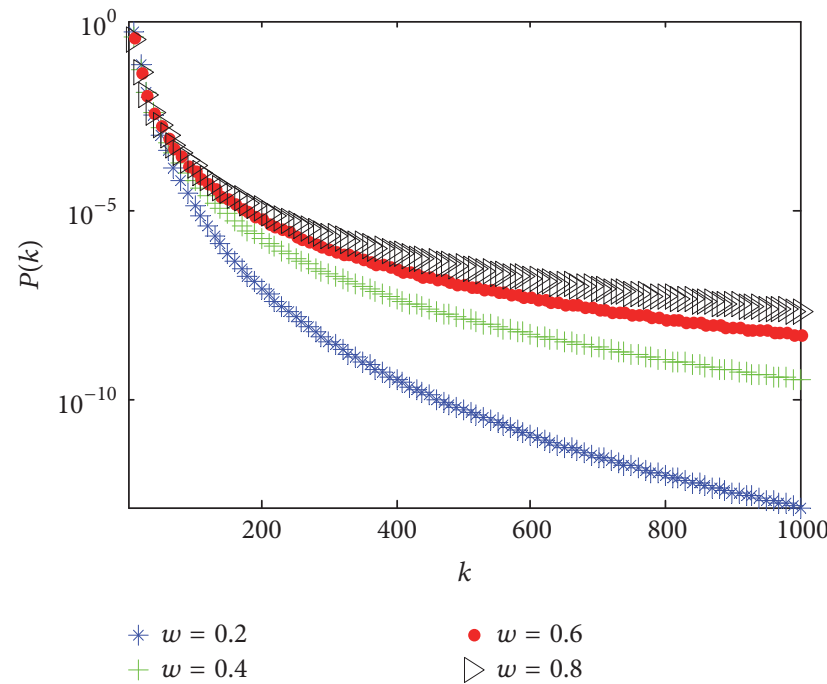

(c)

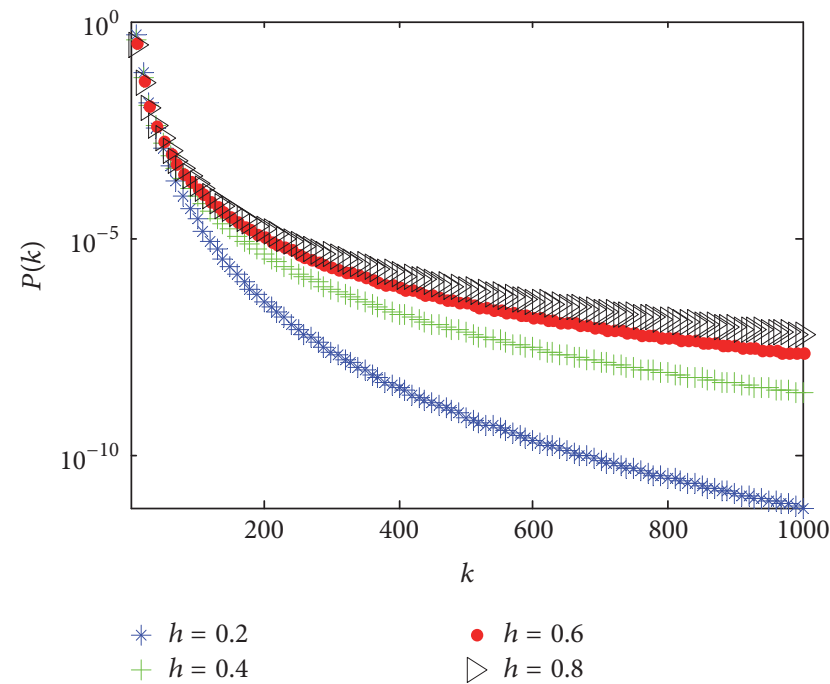

(d)

FIGURE 3: Effect of information transparency on the network topology characteristics of food safety scare behavior diffusion: (a) the network topology characteristics of food safety scare behavior diffusion when the rates of information dissemination are $\lambda=0.2, \lambda=0.4, \lambda=0.6$, and $\lambda=0.8$; (b) the network topology characteristics of food safety scare behavior diffusion when consumer concerns about food safety incidents are $\theta=0.2, \theta=0.4, \theta=0.6$, and $\theta=0.8$; (c) the network topology characteristics of food safety scare behavior diffusion when government food safety supervision information transparency is $w=0.2, w=0.4, w=0.6$, and $w=0.8$; and (d) the network topology characteristics of food safety scare behavior diffusion when media food safety supervision information transparency is $h=0.2, h=0.4, h=0.6$, and $h=0.8$.

namely, the greater the government food safety supervision information transparency and media food safety supervision information transparency are, the smaller the food safety scare behavior forms. The speed of information dissemination and consumers' attention to food safety accidents have "cluster effect" on the network degree distribution of food safety scares behavior diffusion; namely, the greater the information transmission rate is and the higher the number of consumers on food safety accidents is, the more the probability of formation of food safety scares behavior of groups is. Moreover, "cluster effect" is more marked than "scatter effect."
5.2. Analysis of Evolution Characteristics of Food Safety Scare Behavior Diffusion under Different Information Transparency. The impact of evolution characteristics on food safety scare behavior diffusion is simulated (Figures 4 and 5) to describe the evolution characteristics of food safety scare behavior under the effect of information transparency with the different values of the rate of information dissemination $\lambda$, consumer concerns about food safety incidents $\theta$, government food safety supervision information transparency $w$, and media food safety supervision information transparency $h$. The initial values are $\theta=l=\beta=\mu=\delta=0.2, p=\lambda=$ $h=0.3$, and $w=0.4$. 


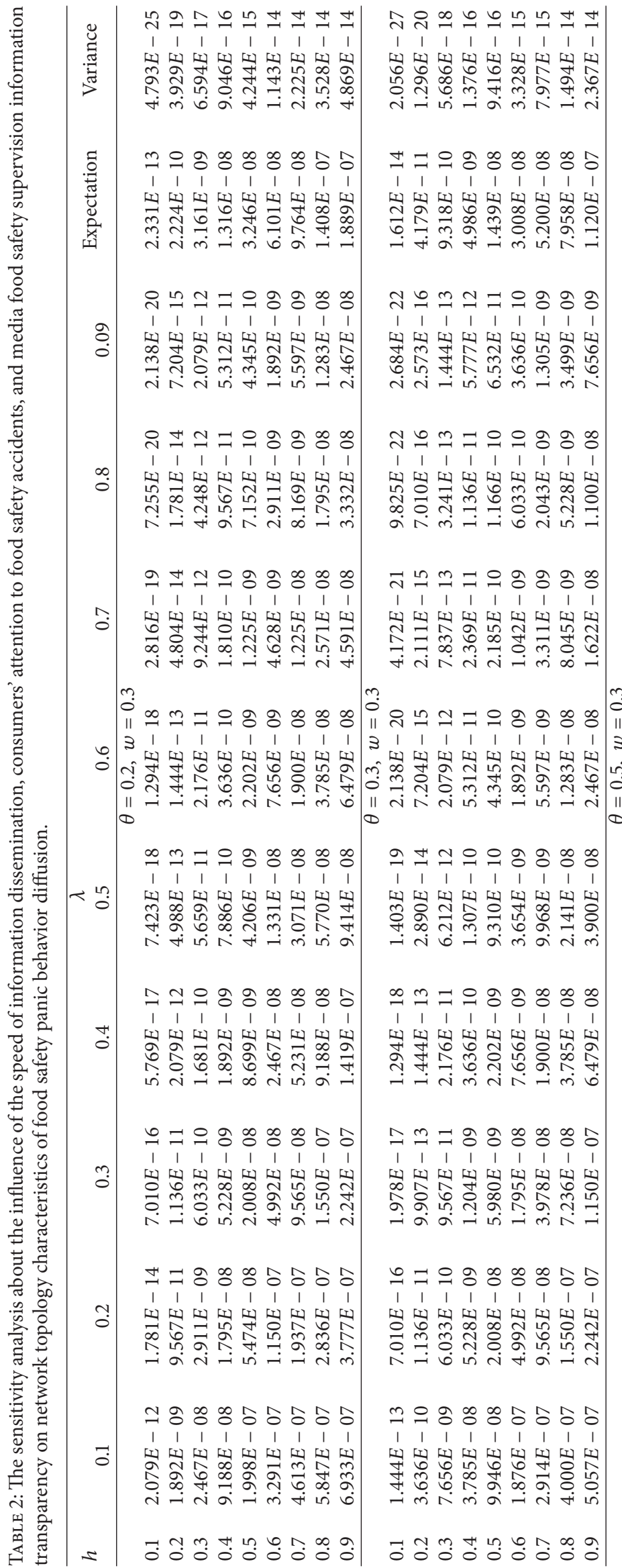

끄음으는 는 는

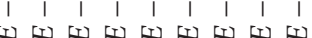

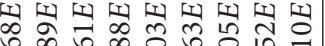
范

눅ㄱㅇㅡ 용

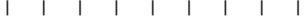

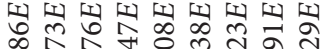

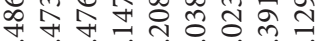

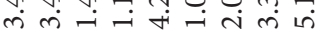

느으ㅁㅡㅡ므그으응

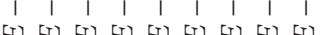

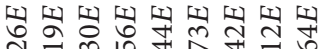
茯

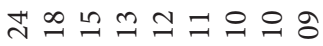

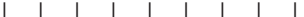

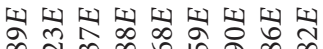
के भु mintini

ㄲㅀ크으응

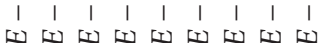

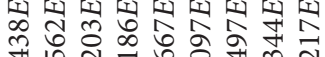

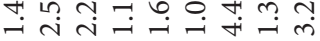
$m e+n=0,0 a$ 그응ㅇㅇ 8 $\begin{array}{llllllllllllllllll}1 & 1 & 1 & 1 & 1 & 1 & 1 & 1 & 1 & 3 & 1 & 1 & 1 & 1 & 1 & 1 & 1 & 1\end{array}$

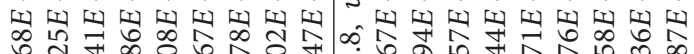

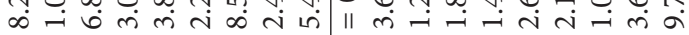

그느그응

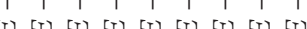

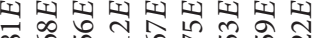

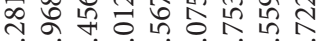
bं 느ㄴㅡㅡ응 $\begin{array}{lllllllll}1 & 1 & 1 & 1 & 1 & 1 & 1 & 1 & 1 \\ 1 & 1 & 1 & 0 & 0 & 0\end{array}$

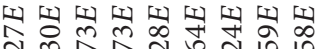
ปิ่ 으보으응 8 \&ᄋ $\begin{array}{lllllllll}1 & 1 & 1 & 1 & 1 & 1 & 1 & 1 & 1\end{array}$

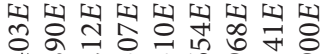

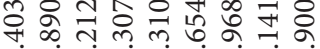
- i

$\stackrel{\infty}{\rightarrow}=$ 응 8 ᄋ $8 \infty$

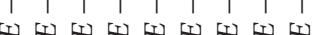

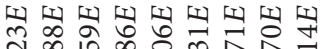
సิ 늨ㅎㅇㅇㅇㅇㅇㅇㅇㅇㅇ

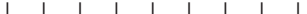

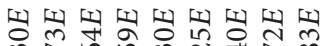
mิ mं F઼

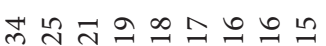

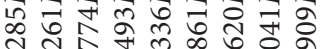

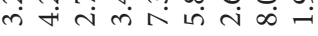

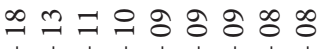

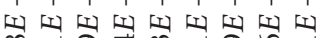
少 ช

오드ㄴㅡㅡㅡㅡㅡㅡㅡ

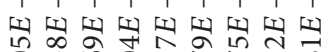

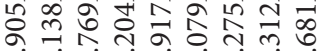
icinta

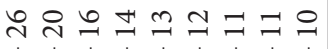

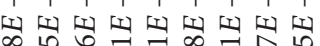

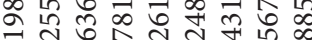
-

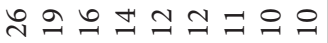

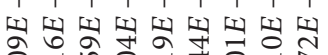
ดे (1)

고그ㄴㅡㅡ긍ㅇㅇㅇㅇ

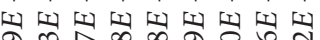
๙

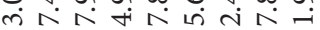

그픜으응 $\begin{array}{lllllllll}1 & 1 & 1 & 1 & 1 & 1 & 1 & 1 & 1 \\ 1 & 1 & 1 & 1\end{array}$

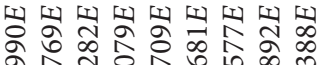
㲾 드ㄴㅡㅡ그응 용

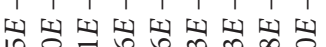
崩号热 बं

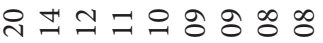

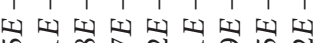

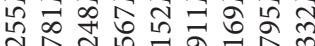
तं

드응ㅇㅇㅇㅇㅇㅇ

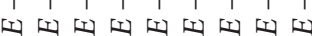

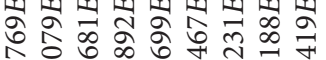
in $i-i_{-1}$ is in

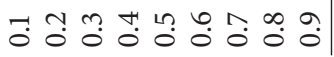

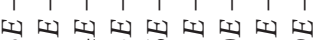




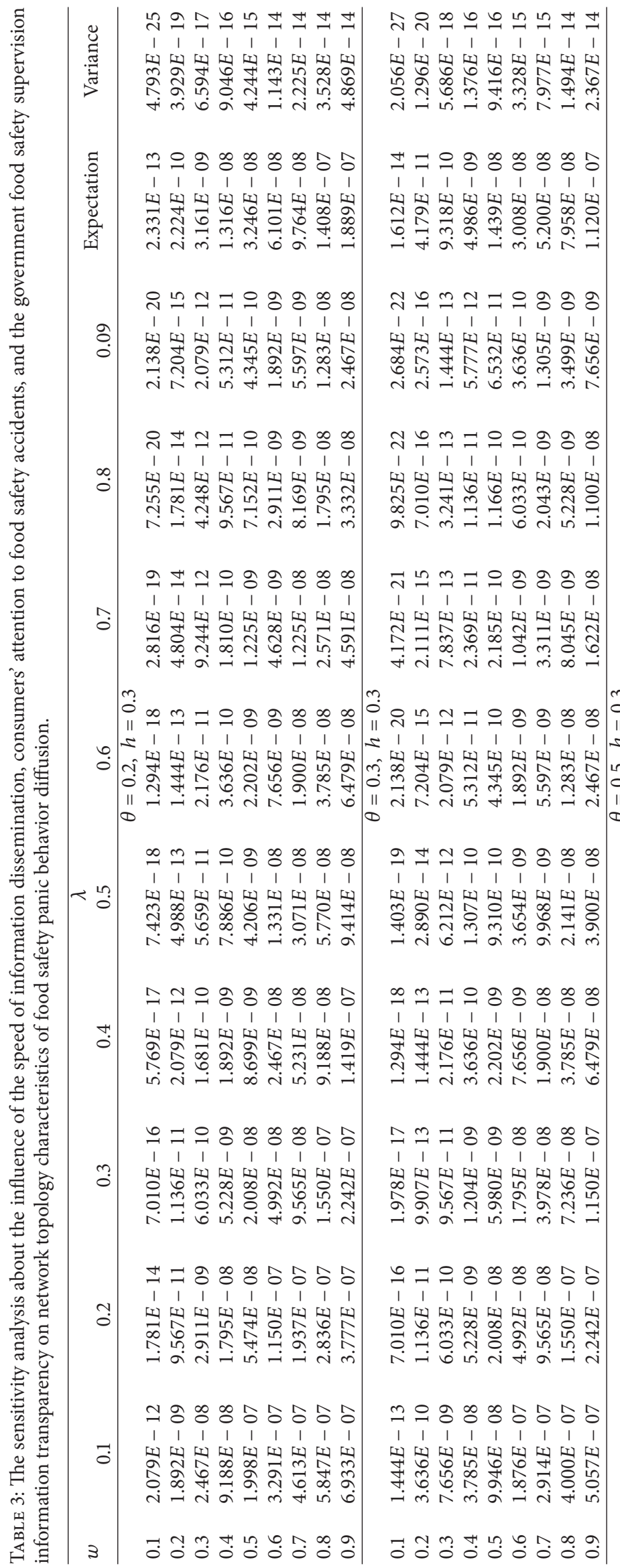

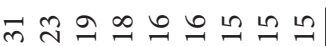
$\begin{array}{lllllllll}1 & 1 & 1 & 1 & 1 & 1 & 1 & 1 & 1 \\ & & & & & \end{array}$

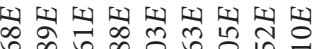

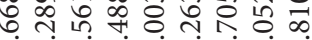
a 0 - $\infty$ in it 누귀응 8 ᄋ

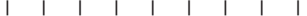

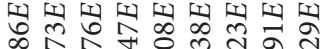

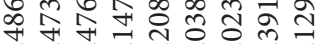

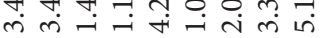

느으므므그으응

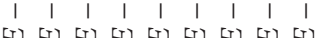

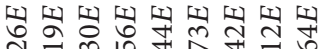
唡学 호느므금응ㅇㅇ $1-1$

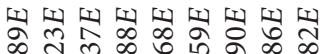

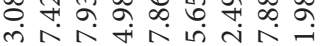
그븜으응ㅇ

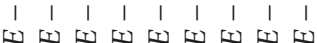

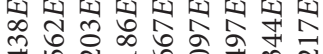
苮 ? 뀨느믕ㅇㅇㅇ

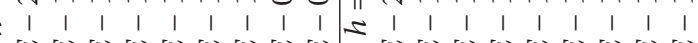

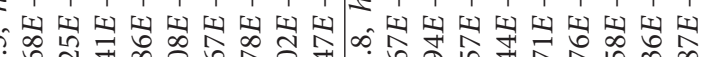
긍 $\infty-i \min m i$ in กํำำ응ำ $\begin{array}{lllllllll} & 1 & 1 & 1 & 1 & 1 & 1 & 1 & 1\end{array}$

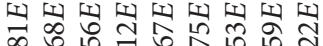

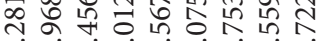
ऊु+ 느ㄴㅡㅡ응

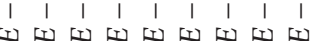

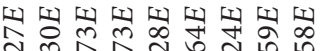
ปิ่ 으복으응 8 $\begin{array}{lllllllll}1 & 1 & 1 & 1 & 1 & 1 & 1 & 1 & 1\end{array}$

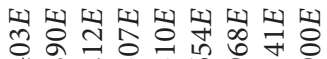

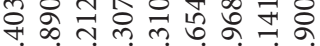
†

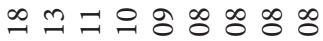
$\begin{array}{lllllllll}1 & 1 & 1 & 1 & 1 & 1 & 1 & 1 & 1 \\ & 1 & 1 & 1 & 1\end{array}$

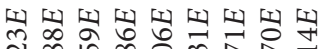
సิ 늨ㅎㅇㅇㅇㅇㅇㅇㅇㅇㅇ $1+1 \quad 1 \quad 1 \quad 1-1 \quad 1-1 \quad 1$

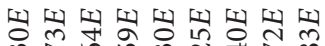
mิ mं F઼

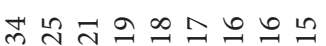

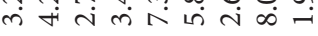

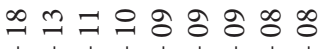

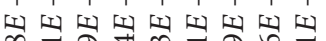

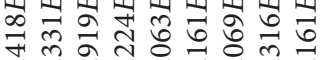
भु

오드ㄴㅡㅡㅡㅡㅡㅡㅡ

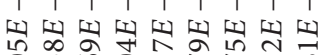

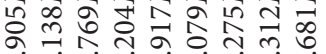
i cintri

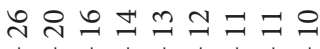

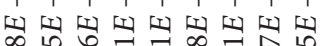
员㤏 -

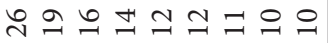

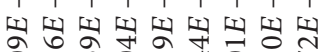
๙ุ

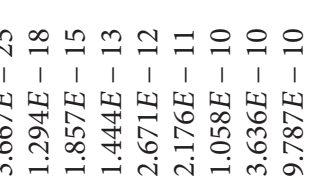
고으므ㄱㅡㅡ으응 1斤毕1

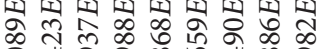
$\infty$ 궁

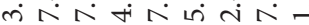

끔크으응 $\begin{array}{ccccccccc}1 & 1 & 1 & 1 & 1 & 1 & 1 & 1 & 1 \\ 1 & 1 & 1 & 1 & 0\end{array}$

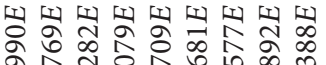
㲾 츤 크으응 옹

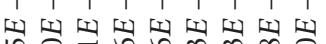
崩 बं

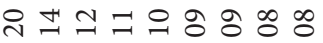
$\begin{array}{lllllllll}1 & 1 & 1 & 1 & 1 & 1 & 1 & 1 & 1 \\ 1 & 1 & 1 & 1 & 1 & 1 & 1 & 0 & 0\end{array}$

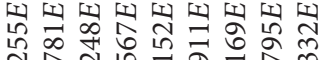

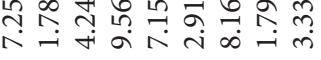
드응ㅇㅇㅇㅇㅇㅇㅇㅇ

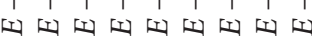

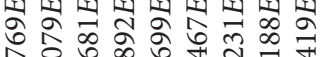
in $i-i_{-1}$ is in

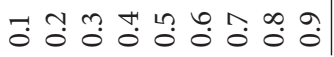

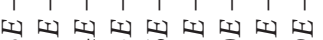

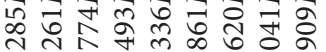




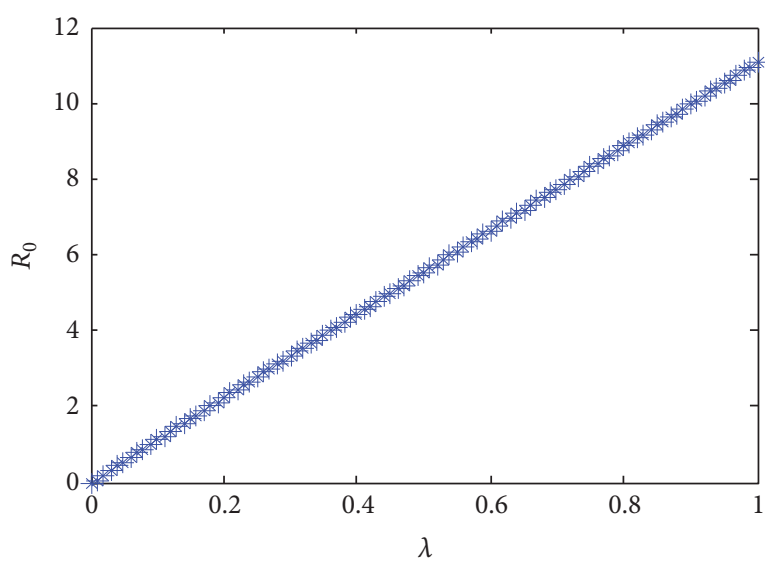

(a)

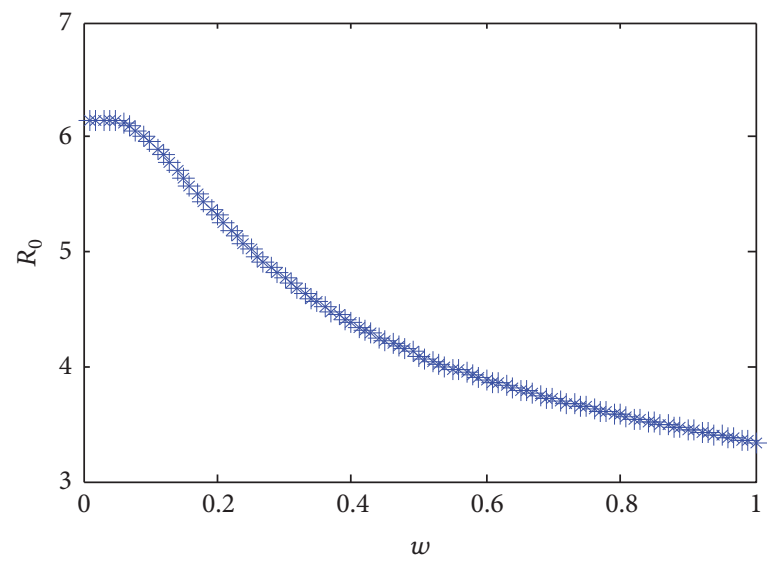

(c)

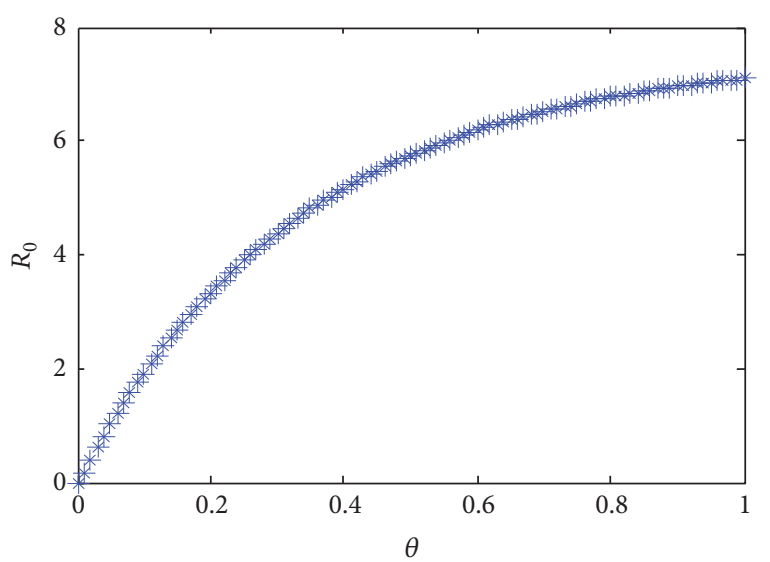

(b)

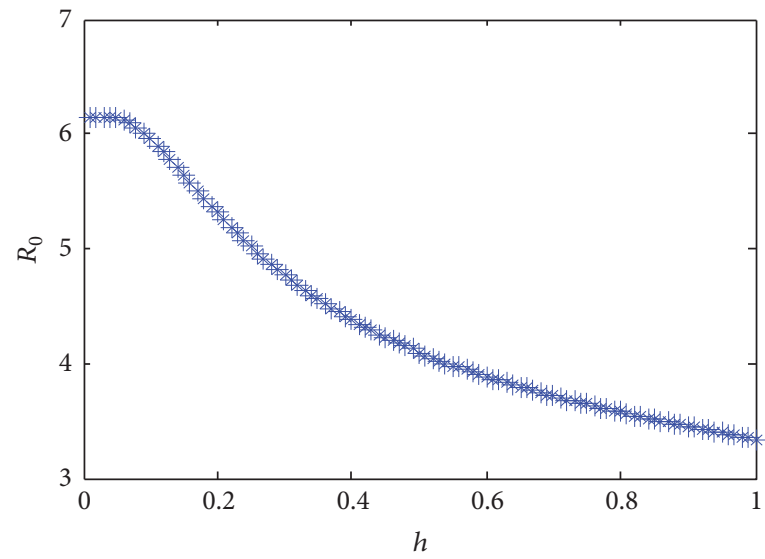

(d)

FIGURE 4: Effect of information transparency on the evolution characteristics of food safety scare behavior diffusion: (a) the effect of the rate of information dissemination $\lambda$ on the evolution characteristics of food safety scare behavior diffusion; (b) the effect of consumer concerns about food safety incidents $\theta$ on the evolution characteristics of food safety scare behavior diffusion; (c) the effect of government food safety supervision information transparency $w$ on the evolution characteristics of food safety scare behavior diffusion; and (d) the effect of media food safety supervision information transparency $h$ on the evolution characteristics of food safety scare behavior diffusion.

The diffusion probability of food safety scare behavior is shown in Figures 4(a) and 4(b). The rate of information dissemination $\lambda$ increases as consumer concerns about food safety incidents $\theta$ increase. The increase of diminishing margins is shown. Figures 4 (a) and 4(b) show that when the rate of information dissemination $\lambda$ and consumer concerns about food safety incidents $\theta$ are less than 0.1 , the basic reproduction number $R_{0}$ is less than 1 , and food safety scare behavior gradually disappears. When the rate of information dissemination $\lambda$ and consumer concerns about food safety incidents $\theta$ are greater than 0.1 , the basic reproduction number $R_{0}$ is more than 1 , and food safety scare behavior diffusion occurs with a nonzero probability. The diffusion probability of food safety scare behavior is high with the increasing rate of information dissemination $\lambda$ and consumer concerns about food safety incidents $\theta$. The diffusion probability of food safety scare behavior shows the declining characteristic of diminishing margins based on Figures 4(c) and 4(d) with increasing government food safety supervision information transparency $w$ and media food safety supervision information transparency $h$. Improving government food safety supervision information transparency $w$ and media food safety supervision information transparency $h$ can reduce the value of the basic reproduction number $R_{0}$. Such improvement can reduce the diffusion probability of food safety scare behavior and play a certain inhibition effect of food safety scare behavior diffusion. However, given a single adjustment of government food safety supervision information transparency $w$ and media food safety supervision information transparency $h$, the value of the basic reproduction number $R_{0}$ remains more than 1 . Therefore, the gradual disappearance of food safety scare behavior cannot be achieved.

Figure 5(a) shows that the diffusion probability of food safety scare behavior is characterized by the increase of diminishing margins with the increase of consumer concerns about food safety incidents $\theta$ and government food safety supervision information transparency $w$. Therefore, the spread of food safety scare behavior is suppressed, and the basic reproduction number $R_{0}$ of less than 1 can be achieved 


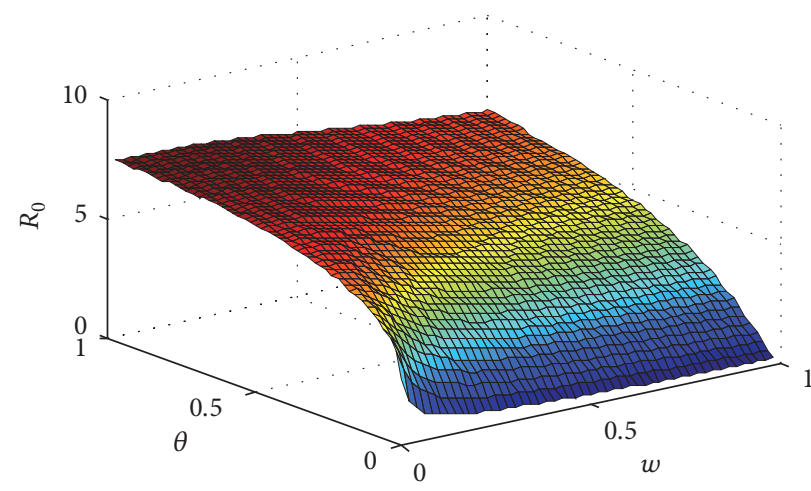

(a)

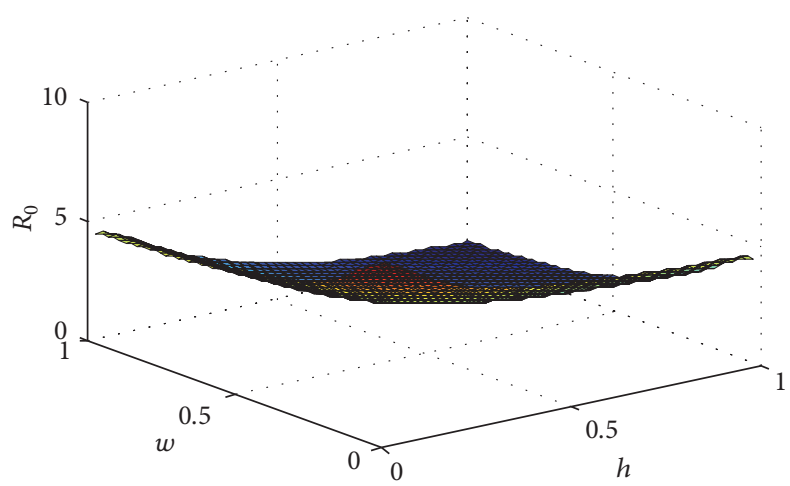

(c)

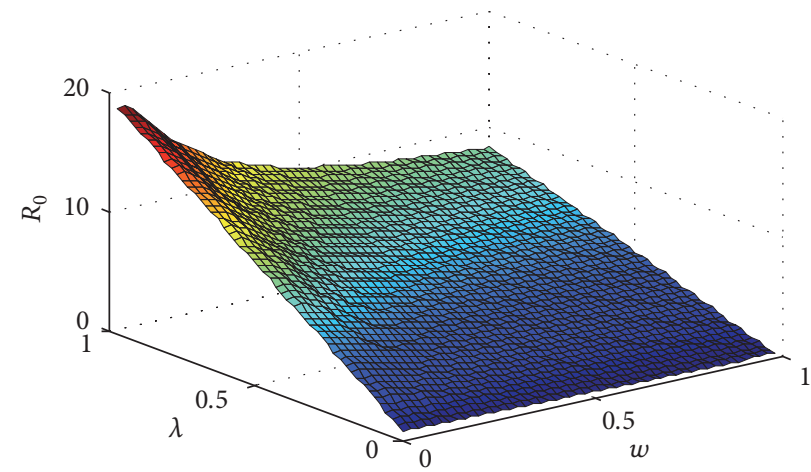

(e)

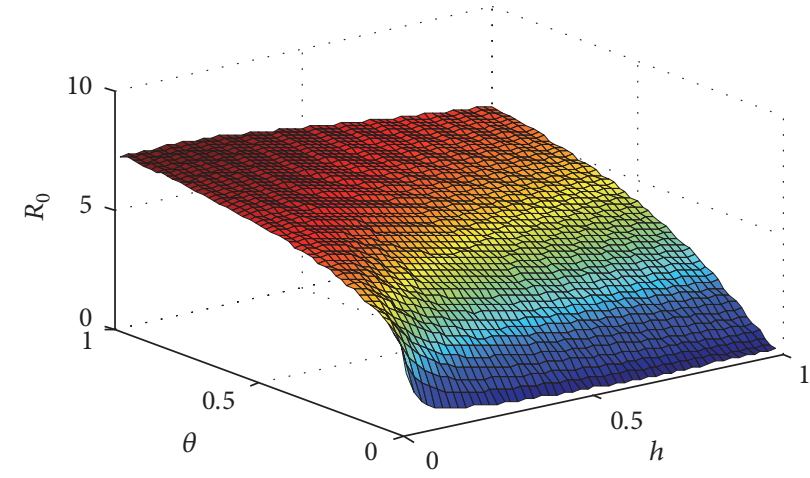

(b)

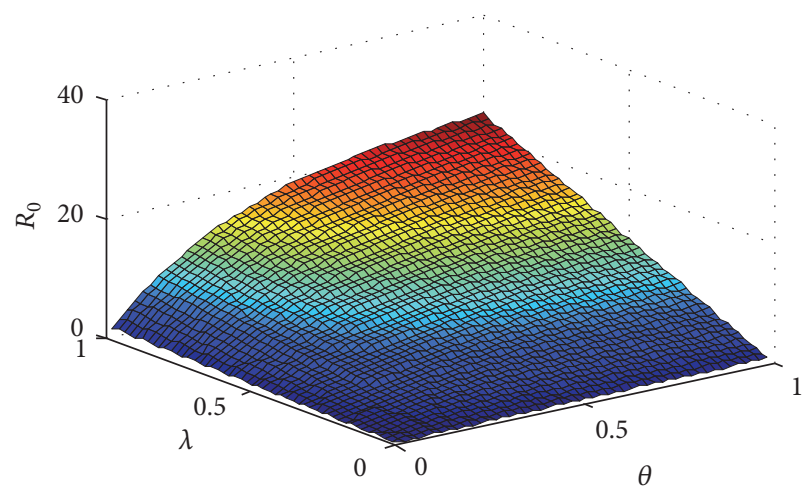

(d)

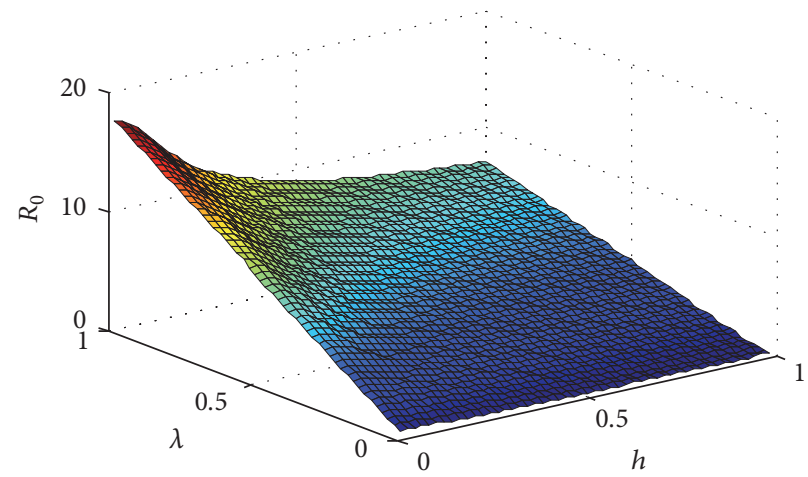

(f)

FIGURE 5: Effect of the interaction of information transparency factors on the evolution characteristics of food safety scare behavior: (a) the effect of the interaction between consumer concerns about food safety incidents $\theta$ and government food safety supervision information transparency $w$ on the evolution characteristics of food safety scare behavior diffusion; (b) the effect of the interaction between consumer concerns about food safety incidents $\theta$ and media food safety supervision information transparency $h$ on the evolution characteristics of food safety scare behavior diffusion; (c) the effect of the interaction between government food safety supervision information transparency $w$ and media food safety supervision information transparency $h$ on the evolution characteristics of food safety scare behavior diffusion; (d) the effect of the interaction between the rate of information dissemination $\lambda$ and consumer concerns about food safety incidents $\theta$ on the evolution characteristics of food safety scare behavior diffusion; (e) the effect of the interaction between the rate of information dissemination $\lambda$ and government food safety supervision information transparency $w$ on the evolution characteristics of food safety scare behavior diffusion; and (f) the effect of the interaction between the rate of information dissemination $\lambda$ and media food safety supervision information transparency $h$ on the evolution characteristics of food safety scare behavior diffusion.

by increasing government food safety supervision information transparency $w$ and decreasing consumer concerns about food safety incidents $\theta$. Figure 5(b) shows that the diffusion probability of food safety scare behavior is characterized by the increase of diminishing margins with the increase of consumer concerns about food safety incidents $\theta$ and media food safety supervision information transparency $h$. Therefore, the purpose of inhibiting the spread of food safety 
scare behavior can be achieved by increasing media food safety supervision information transparency $h$ and reducing consumer concerns about food safety incidents $\theta$. Figure 5 (c) shows that, with the increase of government food safety supervision information transparency $w$ and media food safety supervision information transparency $h$, the diffusion probability of food safety scare behavior shows the decreasing characteristic of diminishing margins. Therefore, curbing the spread of food safety scare behavior can be achieved by improving media food safety supervision information transparency $h$ and government food safety supervision information transparency $w$. Figure 5(d) shows that the diffusion probability of food safety scare behavior is characterized by the increase of diminishing margins with the increase of the rate of information dissemination $\lambda$ and consumer concerns about food safety incidents $\theta$. Therefore, reducing the rate of information dissemination $\lambda$ and consumer concerns about food safety incidents $\theta$ can inhibit the spread of food safety scare behavior. Figure 5(e) shows that, with the increase of the rate of information dissemination $\lambda$ and government food safety supervision information transparency $w$, the diffusion probability of food safety scare behavior is characterized by the increase of diminishing margins. Therefore, reducing the rate of information dissemination $\lambda$ and government food safety supervision information transparency $w$ can inhibit the spread of food safety scare behavior. Figure 5(f) shows that, with the increase of the rate of information dissemination $\lambda$ and media food safety supervision information transparency $h$, the diffusion probability of food safety scare behavior shows an increasing diminishing margin. Therefore, curbing the spread of food safety scare behavior can be achieved by reducing the rate of information dissemination $\lambda$ and increasing media food safety supervision information transparency $h$.

In order to better describe the influence of the speed of information dissemination, consumers' attention to food safety accidents, the government food safety supervision information transparency, and media food safety supervision information transparency on evolution characteristics of food safety panic behavior diffusion, under the circumstance of $l=\beta=\mu=\delta=0.2, m=\eta=5, k=600$, and $p=0.3$, we conduct sensitivity analysis on $\theta, \lambda, w$, and $h$, as shown in Tables 4 and 5 .

The sensitivity analysis of Tables 4 and 5 further verified the conclusions obtained in Figures 4 and 5. And we find that the government food safety supervision information transparency and media food safety supervision information transparency have the "depression effect" on evolutionary existence of the food safety scares behavior diffusion; namely, the greater the government food safety supervision information transparency and media food safety supervision information transparency are, the smaller the diffusion probability of the food safety scare behavior is. The speed of information dissemination and consumers' attention to food safety accidents have "strengthening effect" on the evolutionary existence of the food safety scares behavior diffusion; namely, the greater the information transmission rate is and the higher the number of consumers on food safety accidents is, the more the diffusion probability of food safety scares behavior is. Moreover, "strengthening effect" is more marked than "depression effect." Therefore, in the formulation of the control strategy of food safety scares behavior diffusion, we should focus on the control of transmission rate and reduce the strong attention of consumers on food safety accidents, while improving the government food safety supervision information transparency and media food safety supervision information transparency.

\section{Conclusion}

We construct the network diffusion model of food safety scare behavior, analyze the network topology characteristics of food safety scare behavior diffusion under the effect of information transparency in theory, and analyze the network topology characteristics and evolution characteristics of food safety scare behavior in numerical simulation under different information transparency by introducing information transparency. The theoretical deduction and numerical simulation reveal the following.

(1) Under the effect of consumer concerns about food safety incidents, the rate of information dissemination, media food safety supervision information transparency, and government food safety supervision information transparency, the network degree distribution of food safety scare behavior diffusion shows the declining characteristic of diminishing margins. The significant effects have the following order: government food safety supervision information transparency, consumer concerns about food safety incidents, the rate of information dissemination, and media food safety supervision information transparency. In addition, the sensitivity of the change of the network degree distribution about food safety scare behavior diffusion is enhanced with the increase of information transmission rate and consumer concerns about food safety incidents. The sensitivity of the change of the network degree distribution about food safety scare behavior diffusion reduces with the increase of government food safety supervision information transparency.

(2) The diffusion probability of food safety scare behavior shows the characteristics of monotone increasing, along with the increasing information dissemination rate and consumer concerns about food safety incidents. The increase of government food safety supervision information transparency and media food safety supervision information transparency shows the declining characteristics of diminishing margins. Moreover, the extinction of food safety scare behavior cannot be achieved gradually given a single regulation of government food safety supervision information transparency and media food safety supervision information transparency.

(3) Food safety scare behavior diffusion probability increases, along with the increasing consumer concerns about food safety incidents which show the increasing characteristics of diminishing margins. The increase of government food safety supervision information transparency and media food safety supervision information transparency shows the declining characteristics of diminishing margins. The purpose of inhibiting the spread of food safety scare behavior can be achieved by reducing consumer concerns 


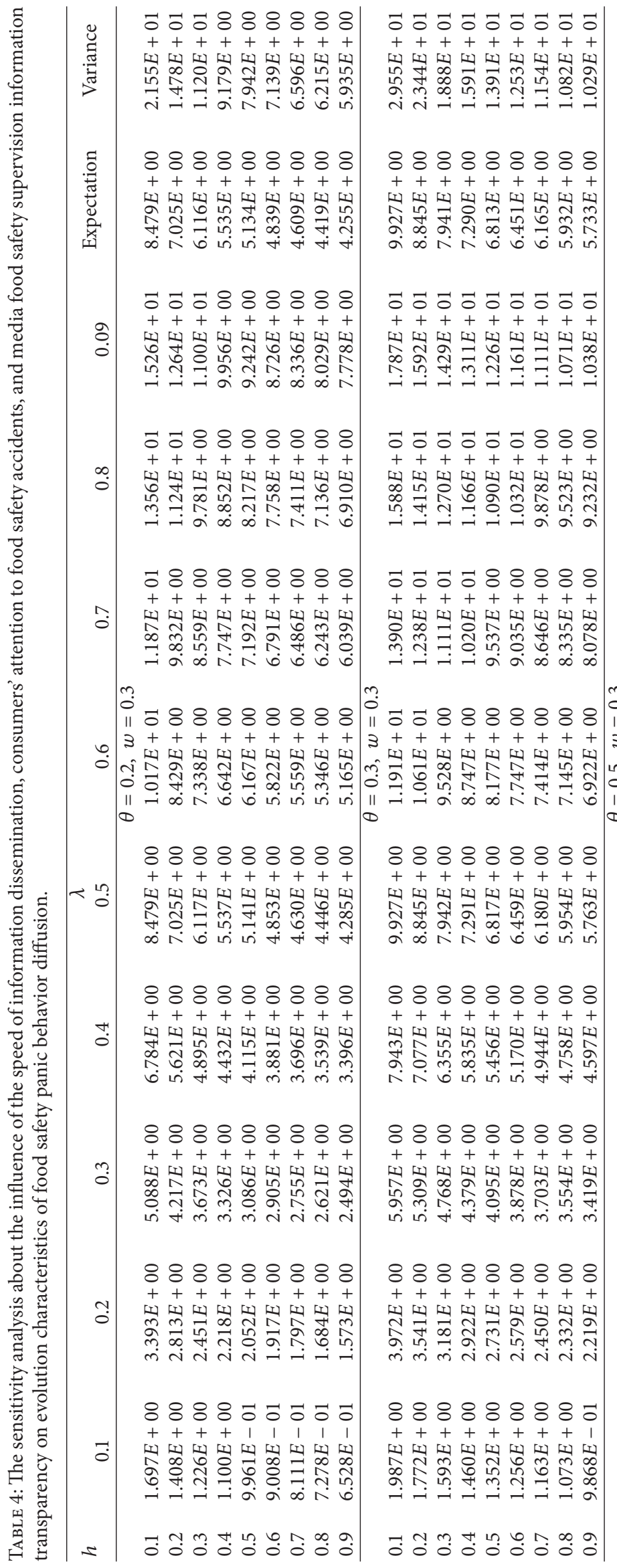

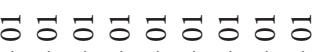
$++++++++$

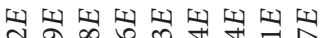
₹

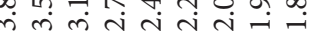

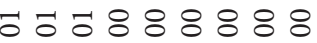
++++++++ +

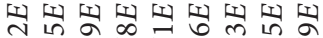
$\Rightarrow$ $\exists-i$ 언

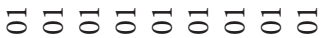
$++++a+t++$

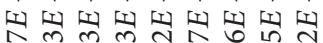

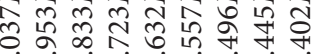
i一r-i-i

จே $+++++++t$

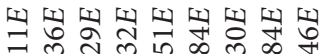
চ

จே $++++++++$

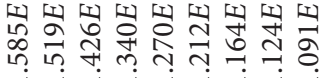
?

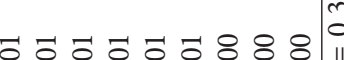
$+++++++++3+++++2+100$

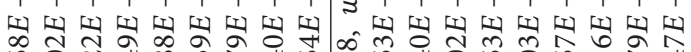

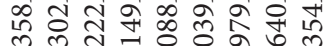
б๐ 0888888 $+++++++++$

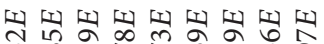

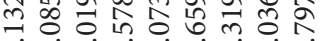

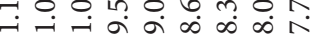
৪৪৪৪৪৪৪৪৪ $++++++++$

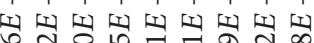

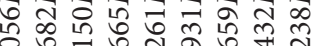

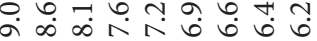
৪৪৪৪৪৪৪৪৪ +++ t t t + + +

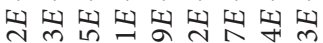

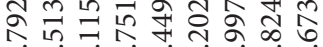

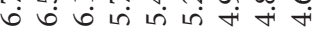

৪৪৪৪৪৪৪৪৪ $+++++4+++$

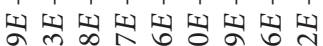

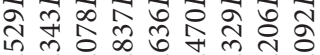

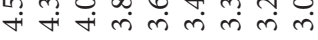
৪৪৪৪৪৪৪৪৪ $++t++$

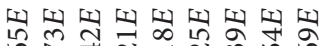

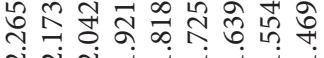
4 i i i r تี

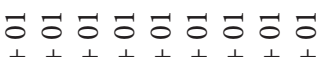

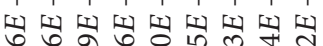

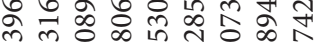

ㅎ๐ㅎㅎㅇ $+4+t+4+t+$

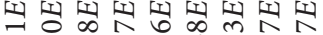

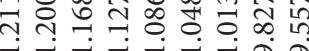
ㅎำ

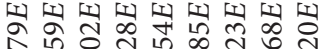

ㅎ๐ㅎㅎㅎ

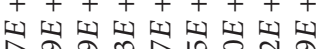

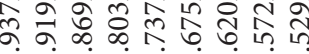

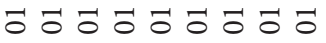
$+++++++++$

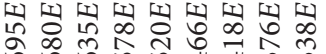
की - - - -

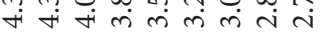
$+++++++t$ i

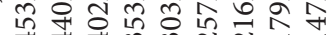
-

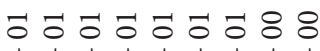
$+++++++1$

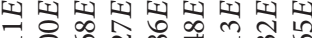
तิ -

৪৪৪৪৪৪৪৪৪ $+++++++++$

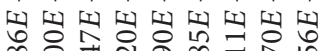

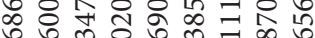

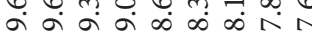

৪৪৪৪৪৪৪৪৪ $++++++++$

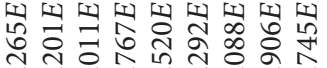

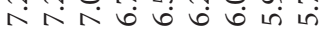

৪৪৪৪৪৪৪৪৪

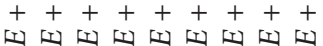
Ұ 

৪৪৪৪৪৪৪৪৪

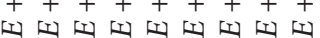

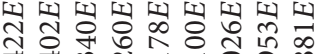

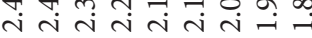

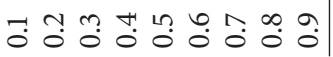




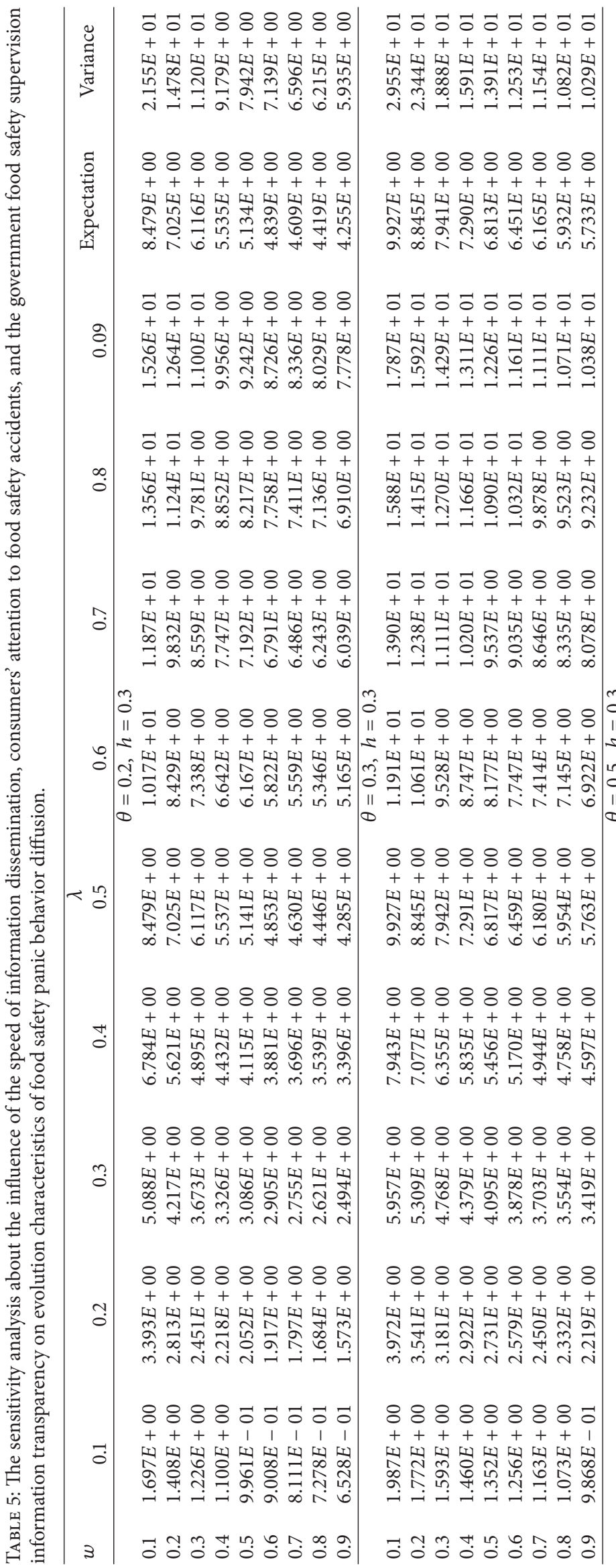

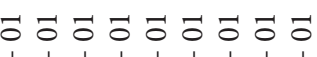

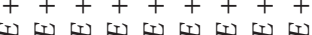

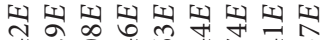

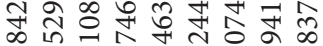

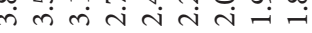
б +++ + + + + + +

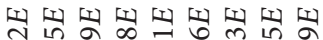

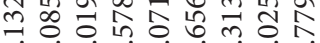

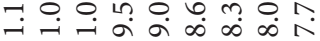

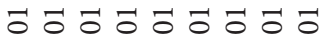
$++++++++t$

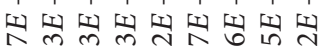

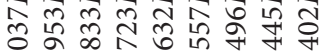
i一 $\rightarrow-\rightarrow-$

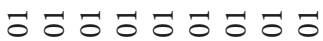
$+++++++t$

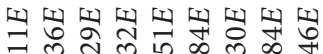

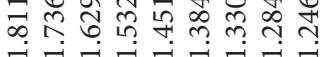
ㅎ๐ㅎㅎㅎㅎ $++++++++$

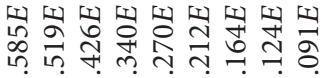
?

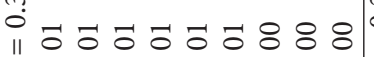
$5+++++++++3+++++++1+4$

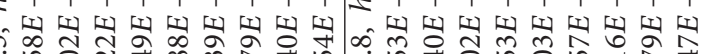

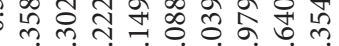

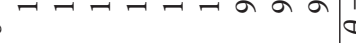
उั5 $+++++++++$

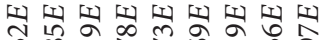

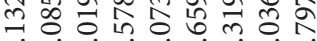

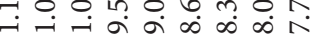
888888888 $+++++++++$

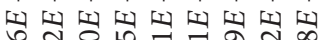

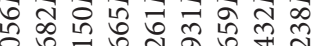

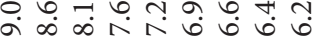
৪৪৪৪৪৪৪৪৪ ++++++ + + +

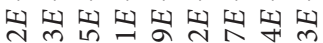
그을

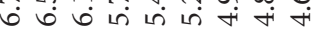

৪৪৪৪৪৪৪৪৪ $+++++++++$

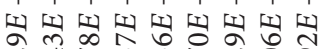

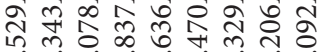

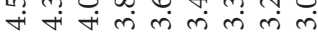
৪৪৪৪৪৪৪৪৪

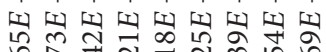

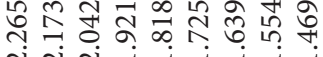
i i i r

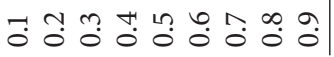

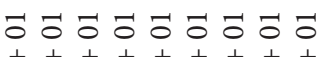

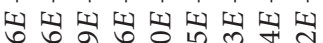

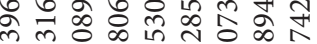

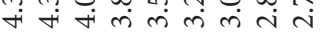

ㅎ๐ㅎㅎㅇ $+7+++$ t + +

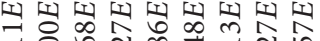

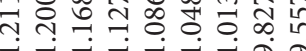
ㅎำ $+++++++t$

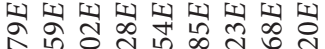
i

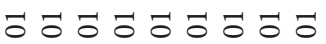

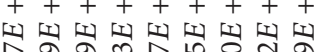

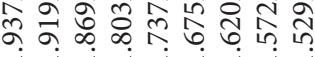

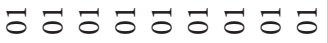
$+++++++++$

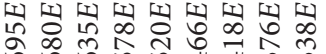
की०

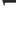

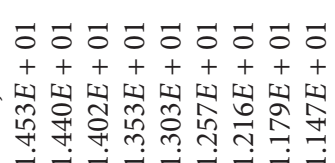
0

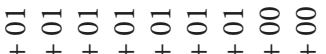

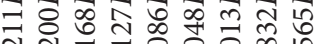
걱

৪৪৪৪৪৪৪৪৪ t + + + + + + + +

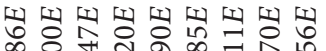
๙a

৪৪৪৪৪৪৪৪৪ + + + + + + + + +

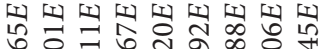

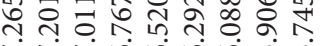
৪৪৪৪৪৪৪৪৪ $+++++++++$

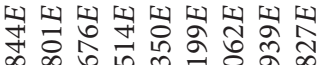
隹品 ৪৪৪৪৪৪৪৪৪

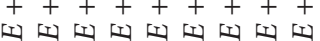

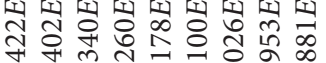
i

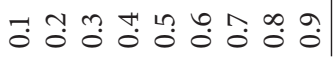

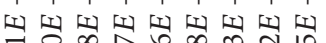


about food safety incidents and improving government food safety supervision information transparency or media food safety supervision information transparency.

(4) Food safety scare behavior diffusion probability increases with government food safety supervision information transparency and media food safety supervision information transparency, which show the declining characteristics of diminishing margins and the increased information dissemination and consumer concerns about food safety incidents, which show the increasing characteristics of diminishing margins. Increasing government food safety supervision information transparency or media food safety supervision information transparency and reducing the rate of information dissemination and consumer concerns about food safety incidents can inhibit food safety scare behavior diffusion.

(5) The diffusion probability of food safety scare behavior presents the increasing characteristics of diminishing margins with the increase of the information transmission rate and government food safety supervision information transparency or media food safety supervision information transparency. The purpose of inhibiting the spread of food safety scare behavior diffusion can be achieved by reducing the rate of information dissemination and improving government food safety supervision information transparency or media food safety supervision information transparency.

(6) The government food safety supervision information transparency and media food safety supervision information transparency have the "scatter effect" on the network degree distribution of food safety scares behavior diffusion. The speed of information dissemination and consumers' attention to food safety accidents have "cluster effect" on the network degree distribution of food safety scares behavior diffusion. And "cluster effect" is more marked than "scatter effect." The government food safety supervision information transparency and media food safety supervision information transparency have the "depression effect" on evolutionary existence of the food safety scares behavior diffusion. The speed of information dissemination and consumers' attention to food safety accidents have "strengthening effect" on the evolutionary existence of the food safety scares behavior diffusion. And "strengthening effect" is more marked than "depression effect."

The present study analyzes the diffusion mechanisms, the network topology characteristics, and the evolution characteristics of the food safety scare behavior from the perspective of information transparency and enriches the research on food safety scare behavior diffusion. Furthermore, the conclusions of this study can provide a powerful theoretical reference for the government to control food safety scare behavior and maintain social stability. However, this study on consumers' food safety scare behavior is from the individual perspectives, rather than from the community, which will be the focus of a follow-up study.

\section{Disclosure}

Tingqiang Chen and Lei Wang are co-first authors.

\section{Conflicts of Interest}

The authors declare that they have no conflicts of interest.

\section{Acknowledgments}

This study is supported by the National Natural Science Foundation of China (nos. 71501094 and 71173103), the Natural Science Foundation of Jiangsu Province of China (no. BK20150961), the Key Project of Philosophy and Social Science Research in Colleges and Universities of Jiangsu Province (2017ZDIXM074), the Outstanding Innovation Team of Philosophy and Social Science Research in Colleges and Universities of Jiangsu Province (2017ZSTD005), and the Qing Lan Project of Jiangsu.

\section{References}

[1] K. G. Grunert, "Food quality and safety: consumer perception and demand," European Review of Agricultural Economics, vol. 32, no. 3, pp. 369-391, 2005.

[2] D. Smith and P. Riethmuller, "Consumer concerns about food safety in Australia and Japan," International Journal of Social Economics, vol. 26, no. 6, pp. 724-742, 1999.

[3] J. M. E. Pennings, B. Wansink, and M. T. G. Meulenberg, "A note on modeling consumer reactions to a crisis: The case of the mad cow disease," International Journal of Research in Marketing, vol. 19, no. 1, pp. 91-100, 2002.

[4] M. L. Roehm and A. M. Tybout, "When will a brand scandal spill over, and how should competitors respond?" Journal of Marketing Research, vol. 43, no. 3, pp. 366-373, 2006.

[5] J. de Jonge, L. Frewer, H. Van Trijp, R. Jan Renes, W. de Wit, and J. Timmers, "Monitoring consumer confidence in food safety: An exploratory study," British Food Journal, vol. 106, pp. 837849, 2004.

[6] B. Kealesitse and I. O. Kabama, "Exploring the influence of quality and safety on consumers' food purchase decisions in Botswana," International Journal of Business Administration, vol. 3, no. 2, pp. 90-97, 2012.

[7] A. G. Long, J. J. Kastner, and R. Kassatly, "Is food security a new tariff? Explaining changes in sanitary and phytosanitary regulations by world trade organization members," Global Economy Journal, vol. 13, no. 1, pp. 25-46, 2013.

[8] P. Fousekis and J. Revell B, "Meat demand in the UK: A Differential Approach," Journal of Agricultural \&Applied Economics, vol. 32, no. 1, pp. 11-19, 2000.

[9] A. M. Angulo, J. M. Gil, and L. Tamburo, "Food safety and consumers' willingness to pay for labelled beef in Spain," Journal of Food Products Marketing, vol. 11, no. 3, pp. 89-105, 2005.

[10] G. T. Tonsor, "Consumer inferences of food safety and quality," European Review of Agricultural Economics, vol. 38, no. 2, pp. 213-235, 2011.

[11] R. Alphonce and F. Alfnes, "Consumer willingness to pay for food safety in Tanzania: An incentive-aligned conjoint analysis," International Journal of Consumer Studies, vol. 36, no. 4, pp. 394-400, 2012.

[12] T. Li, J. C. Bernard, Z. A. Johnston, K. D. Messer, and H. M. Kaiser, "Consumer preferences before and after a food safety scare: An experimental analysis of the 2010 egg recall," Food Policy, vol. 66, pp. 25-34, 2017. 
[13] Y. Rmw and J. Morris, "Consumer perception of food risk in chicken meat," Nutrition \& Food Science, vol. 31, no. 6, pp. 270279, 2001.

[14] D. Mahon and C. Cowan, "Irish consumers' perception of food safety risk in minced beef," British Food Journal, vol. 106, no. 4, pp. 301-312, 2004.

[15] S. A. Hornibrook, M. McCarthy, and A. Fearne, "Consumers' perception of risk: The case of beef purchases in Irish supermarkets," International Journal of Retail \& Distribution Management, vol. 33, no. 10, pp. 701-715, 2005.

[16] J. Polimeni M, R. Iorgulescu I, and M. Balan, "Food safety, food security and environmental risks," Internal Auditing \& Risk Management, vol. 1, no. 29, pp. 53-67, 2013.

[17] J. Wang and T. Chen, "The spread model of food safety risk under the supply-demand disturbance," SpringerPlus, vol. 5, no. $1,2016$.

[18] J. A. Caswell and E. M. Mojduszka, "Using informational labeling to influence the market for quality in food products," American Journal of Agricultural Economics, vol. 78, no. 5, pp. 1248-1253, 1996.

[19] C. Ritson and L. W. Mai, "The economics of food safety," Nutrition \& Food Science, vol. 98, no. 5, pp. 253-259, 1998.

[20] T. Chen, L. Wang, and J. Wang, "Transparent assessment of the supervision information in China's food safety: A fuzzy-ANP comprehensive evaluation method," Journal of Food Quality, vol. 2017, Article ID 4340869, 14 pages, 2017.

[21] T. Chen, B. Ma, and J. Wang, "SIRS contagion model of food safety risk," Journal of Food Safety, p. e12410.

[22] Y. Liu, B. Wang, B. Wu, S. Shang, Y. Zhang, and C. Shi, "Characterizing super-spreading in microblog: an epidemicbased information propagation model," Physica A: Statistical Mechanics and its Applications, vol. 463, pp. 202-218, 2016.

[23] G. Zaman, Y. H. Kang, G. Cho, and I. H. Jung, "Optimal strategy of vaccination \& treatment in an SIR epidemic model," Mathematics and Computers in Simulation, vol. 136, pp. 63-77, 2017.

[24] R. N. Carter and S. D. Prince, "Epidemic models used to explain biogeographical distribution limits," Nature, vol. 293, no. 5834, pp. 644-645, 1981.

[25] P.-A. Noël, B. Davoudi, R. C. Brunham, L. J. Dubé, and B. Pourbohloul, "Time evolution of epidemic disease on finite and infinite networks," Physical Review E: Statistical, Nonlinear, and Soft Matter Physics, vol. 79, article 026101, no. 2, 2009.

[26] Y. Tong G, W. Shi F, D. Liu et al., "Genetic diversity and evolutionary dynamics of Ebola virus in Sierra Leone," Nature, vol. 524, no. 7563, 93 pages, 2015.

[27] J. Rode and A. Weber, "Does localized imitation drive technology adoption? A case study on rooftop photovoltaic systems in Germany," Journal of Environmental Economics and Management, vol. 78, pp. 38-48, 2016.

[28] T.-Q. Chen and J.-M. He, "A network model of credit risk contagion," Discrete Dynamics in Nature and Society, vol. 2012, Article ID 513982, 13 pages, 2012.

[29] X. Feng and H. Hu, "Measurement and internalization of systemic risk in a global banking network," International Journal of Modern Physics C, vol. 24, article 1250093, no. 1, 2013.

[30] A. Derbali and S. Hallara, "Measuring systemic risk of Greek banks: New approach by using the epidemic model "SEIR"," Cogent Business \& Management, vol. 3, article 1153864, no. 1, 2016.
[31] V. Giorno and S. Spina, "Rumor spreading models with random denials," Physica A: Statistical Mechanics and its Applications, vol. 461, pp. 569-576, 2016.

[32] S. Hosseini and M. A. Azgomi, "A model for malware propagation in scale-free networks based on rumor spreading process," Computer Networks, vol. 108, pp. 97-107, 2016.

[33] V. Colizza and A. Vespignani, "Epidemic modeling in metapopulation systems with heterogeneous coupling pattern: theory and simulations," Journal of Theoretical Biology, vol. 251, no. 3, pp. 450-467, 2008.

[34] F. Zhang, L. Lu I, and H. Yu X, "Survey of transmission models of infectious diseases," System Engineering Theory \& Practice, vol. 31, no. 9, pp. 1763-1744, 2011.

[35] S. Li and Z. Jin, "Dynamic modeling and analysis of sexually transmitted diseases on heterogeneous networks," Physica A: Statistical Mechanics and its Applications, vol. 427, pp. 192-201, 2015.

[36] J. Skaza and B. Blais, "Modeling the infectiousness of Twitter hashtags," Physica A: Statistical Mechanics and its Applications, vol. 465, pp. 289-296, 2017.

[37] R. Pastor-Satorras and A. Vespignani, "Epidemics and immunization in scale-free networks," in Handbook of Graph \& Networks, S. Bornholdt and H. G. Schuster, Eds., pp. 111-130, 2002.

[38] R. E. Lofstedt, "How can we make food risk communication better: Where are we and where are we going?" Journal of Risk Research, vol. 9, no. 8, pp. 869-890, 2006.

[39] P. J. Williamson, "Sales and Service Strategy for the Single European Market," Business Strategy Review, vol. 3, no. 2, pp. 17-43, 1992.

[40] M. Mazzocchi, A. Lobb, W. B. Traill, and A. Cavicchi, "Food scares and trust: A European study," Journal of Agricultural Economics, vol. 59, no. 1, pp. 2-24, 2008.

[41] S. Cope, L. J. Frewer, J. Houghton, G. Rowe, A. R. H. Fischer, and J. de Jonge, "Consumer perceptions of best practice in food risk communication and management: Implications for risk analysis policy," Food Policy, vol. 35, no. 4, pp. 349-357, 2010.

[42] L. Austin, B. F. Liu, and Y. Jin, "How Audiences Seek Out Crisis Information: Exploring the Social-Mediated Crisis Communication Model," Journal of Applied Communication Research, vol. 40, no. 2, pp. 188-207, 2012.

[43] M. E. Gilpin and F. G. Ayala, "Global models of growth and competition," Proceedings of the National Acadamy of Sciences of the United States of America, vol. 70, pp. 3590-3593, 1973.

[44] Y. Moreno, R. Pastor-Satorras, and A. Vespignani, "Epidemic outbreaks in complex heterogeneous networks," The European Physical Journal B, vol. 26, no. 4, pp. 521-529, 2002.

[45] M. Boguá, R. Pastorsatorras, and A. Vespignani, "Epidemic spreading in complex networks with degree correlations," Lecture Notes in Physics, vol. 625, no. 3, pp. 331-348, 2003.

[46] R. Yang, B.-H. Wang, J. Ren et al., "Epidemic spreading on heterogeneous networks with identical infectivity," Physics Letters A, vol. 364, no. 3-4, pp. 189-193, 2007.

[47] T. Chen, J. He, and Q. Yin, "Dynamics evolution of credit risk contagion in the CRT market," Discrete Dynamics in Nature and Society, vol. 2013, Article ID 206201, 9 pages, 2013.

[48] M. R. Anderson and R. M. May, Infectious Diseases of Humans, Oxford University Press, Oxford, UK, 1991.

[49] T. Chen, X. Li, and J. Wang, "Spatial interaction model of credit risk contagion in the CRT market," Computational Economics, vol. 46, no. 4, pp. 519-537, 2015. 


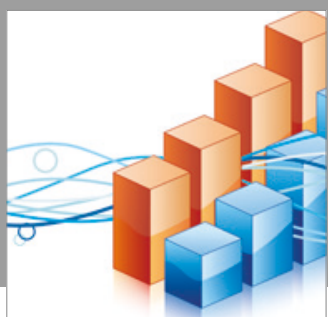

Advances in

Operations Research

vatersals

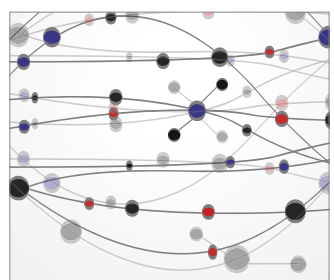

\section{The Scientific} World Journal
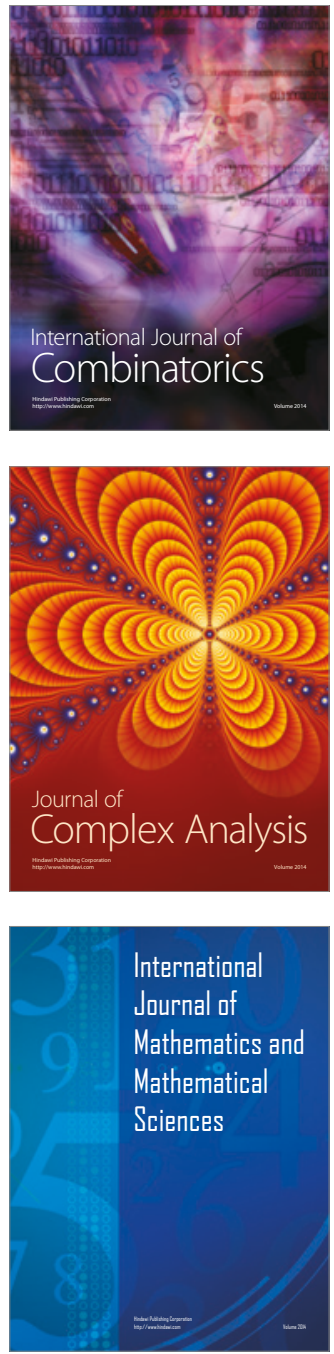
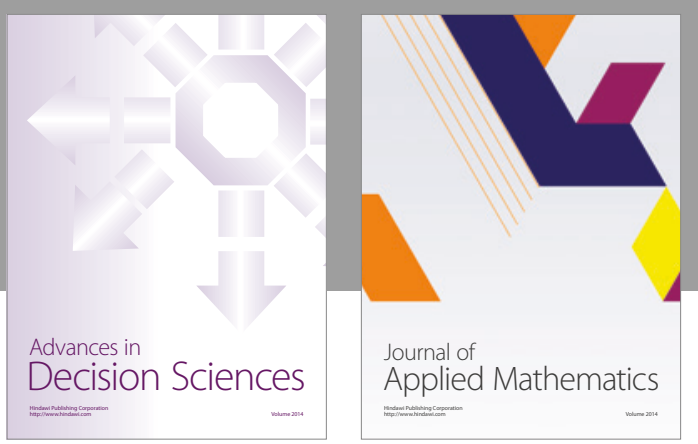

Algebra

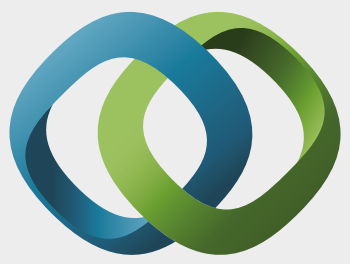

\section{Hindawi}

Submit your manuscripts at

https://www.hindawi.com
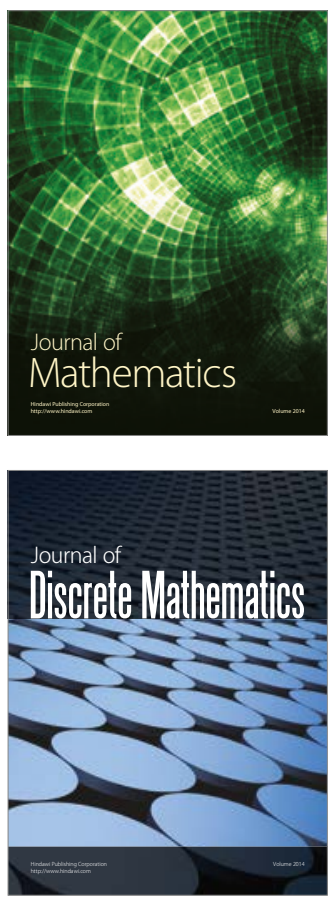

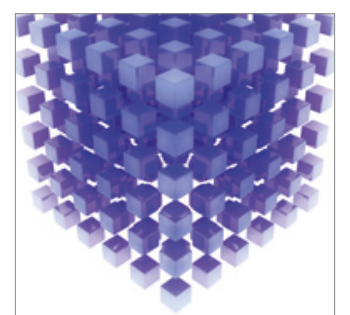

Mathematical Problems in Engineering
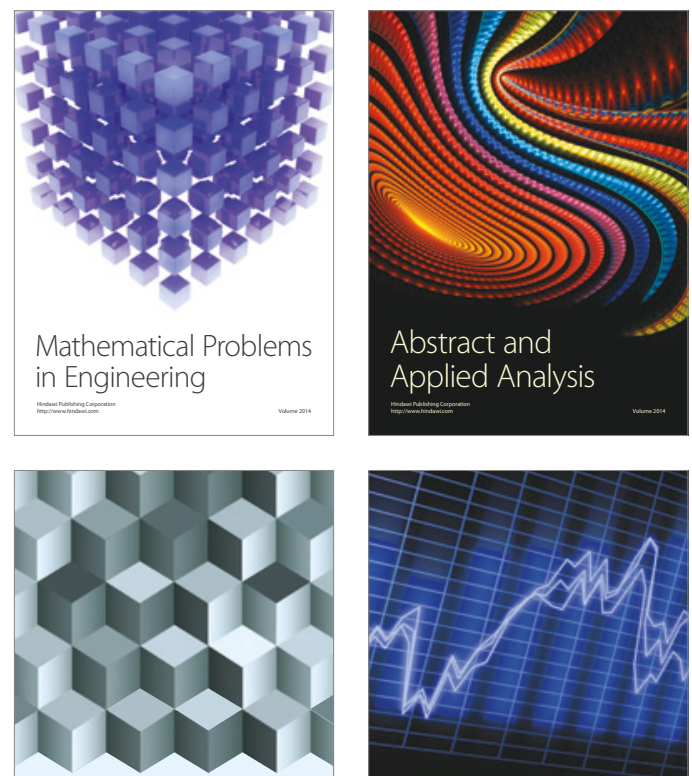

Journal of

Function Spaces

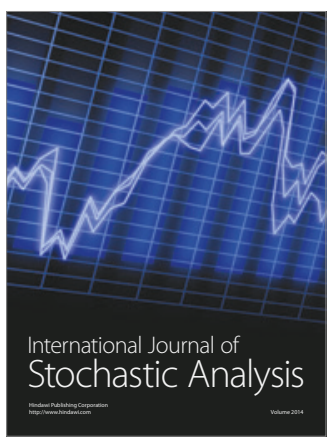

Probability and Statistics
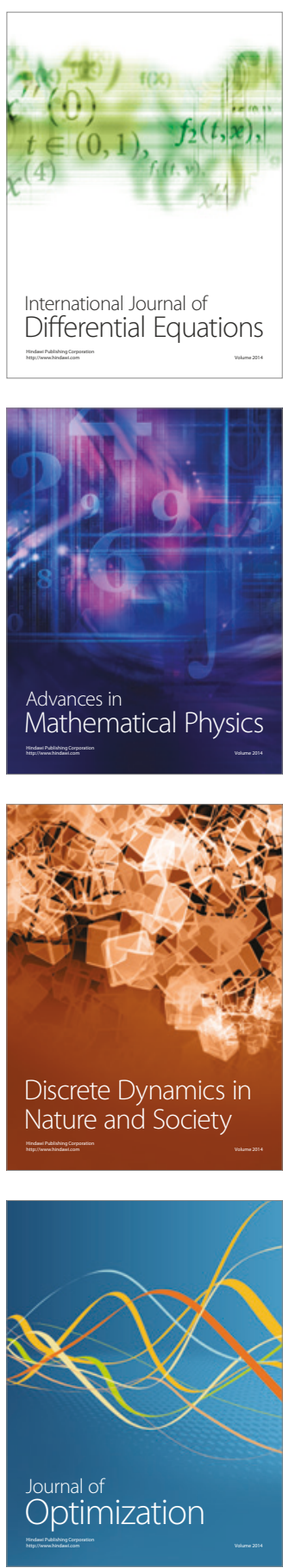